OPEN ACCESS

Edited by:

Guillermo A. Mena Marugán,

Instituto de Estructura de la Materia

(IEM), Spain

Reviewed by:

Jorge Pullin,

Louisiana State University,

United States

Mercedes Martin-Benito,

Complutense University of Madrid,

Spain

Jerónimo Cortez,

Universidad Nacional Autónoma de

México, Mexico

${ }^{*}$ Correspondence:

Abhay Ashtekar

ashtekar.gravity@gmail.com

Specialty section:

This article was submitted

to Cosmology,

a section of the journa

Frontiers in Astronomy and Space

Sciences

Received: 24 March 2021 Accepted: 28 April 2021

Published: 04 June 2021

Citation:

Ashtekar A, Gupt B and Sreenath V (2021) Cosmic Tango Between the

Very Small and the Very Large: Addressing CMB Anomalies Through

Loop Quantum Cosmology.

Front. Astron. Space Sci. 8:685288.

doi: 10.3389/fspas.2021.685288

\section{Cosmic Tango Between the Very Small and the Very Large: Addressing CMB Anomalies Through Loop Quantum Cosmology}

\author{
Abhay Ashtekar ${ }^{1 *}$, Brajesh Gupt ${ }^{1,2}$ and V. Sreenath ${ }^{3}$ \\ ${ }^{1}$ Institute for Gravitation and the Cosmos and Physics Department, The Pennsylvania State University, University Park, \\ Pennsylvania, PA, United States, ${ }^{2}$ Texas Advanced Computing Center, The University of Texas at Austin, Austin, TX, \\ United States, ${ }^{3}$ Department of Physics, National Institute of Technology Karnataka, Surathkal, India
}

While the standard, six-parameter, spatially flat $\Lambda$ CDM model has been highly successful, certain anomalies in the cosmic microwave background bring out a tension between this model and observations. The statistical significance of any one anomaly is small. However, taken together, the presence of two or more of them imply that according to standard inflationary theories we live in quite an exceptional Universe. We revisit the analysis of the PLANCK collaboration using loop quantum cosmology, where an unforeseen interplay between the ultraviolet and the infrared makes the primordial power spectrum scale dependent at very small $k$. Consequently, we are led to a somewhat different $\Lambda \mathrm{CDM}$ Universe in which anomalies associated with large scale power suppression and the lensing amplitude are both alleviated. The analysis also leads to new predictions for future observations. This article is addressed both to cosmology and loop quantum gravity communities, and we have attempted to make it self-contained.

Keywords: CMB, anomalies, loop quantum cosmology (LQC), big bounce, UV-IR interplay

\section{INTRODUCTION}

The quantum geometry effects underlying loop quantum gravity (LQG) lead to a natural resolution of the big bang singularity [see, e.g. Ashtekar and Singh (2004), Agullo and Singh (2017), for reviews]. Therefore, one can hope to meaningfully extend the standard inflationary paradigm to the Planck regime. Over the past decade, several closely related approaches have been used to carry out this task, leading to a striking interplay between theory and observations [see, in particular Agullo et al. (2012), Agullo et al. (2013a), Agullo et al. (2013b), Fernandez-Mendez et al. (2012), Barrau et al. (2014), Linsefors et al. (2013), Ashtekar and Barrau (2015), Agullo and Morris (2015), Agullo (2015), Ashtekar and Gupt (2017a), Ashtekar and Gupt (2017b), Gomar et al. (2017), Agullo et al. (2018), Barrau et al. (2018), Agullo et al. (2020a), Agullo et al. (2020b), Sreenath et al. (2019), Agullo et al. (2021a), Agullo et al. (2021b)]. In this article we will focus on the recent results that shed new light on the anomalous features seen in the cosmic microwave background (CMB). Specifically, we will show that in our approach two of the anomalies seen in the CMB can be accounted for using the preinflationary dynamics of loop quantum cosmology (LQC). This phase of dynamics alters the quantum state of cosmological perturbations at the onset of the (relevant part of the) slow roll, leading to revised values of the six parameters that characterize the $\Lambda$ CDM Universe. The revision alleviates the tension due to two anomalies that have received considerable attention, while leaving the successes of standard inflation intact. Main results were reported in Ashtekar et al. (2020). The 
purpose of this paper is to provide details and also present some supplementary material to put the results in a broader context. These results illustrate that LQC has matured sufficiently to lead to testable predictions.

The paper is addressed both to the LQG community and cosmologists. For the benefit of the LQG community, that primarily focuses on mathematical physics, we have included a discussion of the interplay between theory and observations that leads to the six parameter $\Lambda C D M$ cosmological model. We will summarize the underlying procedure and point out certain subtleties in data analysis. For cosmologists, we will summarize the key features of LQC that lead to new observable predictions. Specifically we will explain how the quantum geometry effects in the ultraviolet, that lead to the singularity resolution, have unforeseen and interesting consequences on the dynamics of cosmological perturbations in the infrared. It is this 'cosmic tango' between the very small and the very large that alleviates anomalies. Overall, in terms of conceptual flow, we have attempted to make this paper selfcontained. In particular, within the page limits of this special issue, we clarify apparently conflicting statements in the LQC literature. In order to make the material accessible to both communities, we will have to briefly review ideas and results that are likely to be well-known in one community but not the other.

The two anomalies we focus on arise as follows. Motivated in large part by inflationary scenarios, the CMB analysis generally begins by assuming that the primordial scalar power spectrum has a nearly scale invariant form, characterized by just two parameters, the scalar perturbation amplitude $A_{s}$ and the scalar spectral index $n_{s}$. We will refer to this form as the standard ansatz (SA). $A_{s}, n_{s}$ and 4 other parameters (discussed in Section 2.1) characterize a specific $\Lambda$ CDM Universe. Given these six parameters one can evolve the primordial perturbations using known astrophysics and predict the observable power spectra. By varying the values of the 6 parameters, and confronting the theoretical prediction with observations, one finds the posterior probability distributions of the six cosmological parameters. By and large the $\mathrm{CMB}$ observations can be well explained using the $\Lambda$ CDM Universe determined by the marginalized mean values of these parameters. However, one also finds some anomalous features. The first is power suppression at large angular scales: the observed power in the temperature-temperature (TT) spectrum is suppressed for $\ell \leq 30$ in the spherical harmonic decomposition, relative to what the theory predicts. The second anomaly we focus on is associated with the so-called lensing amplitude, $A_{L}$, associated with gravitational lensing that the $\mathrm{CMB}$ photons experience as they propagate from the surface of last scattering to us. The $\Lambda$ CDM cosmology based on the $S A$ assumes $A_{L}=1$ while, when it is allowed to vary, $A_{L}$ prefers a value larger than unity. This tension hints at an internal inconsistency. To alleviate it, one can introduce a positive spatial curvature (Handley, 2019) but then there are inconsistencies with the low $z$ measurements, prompting a recent suggestion (Di Valentino et al., 2019) that this anomaly gives rise to a "possible crisis in cosmology."
As we will see, both the anomalies are simultaneously alleviated in our approach. The key new element is the following: Pre-inflationary dynamics of LQC leads to a primordial power spectrum that differs from the $S A$, but only at large angular scales. While it continues to be nearly scale invariant-and essentially indistinguishable from the one given by the $S A$-for $\ell \gtrsim 30$, there is a specific power suppression for $\ell \leqq 30$. As a result, the best-fit values of the six cosmological parameters change. Interestingly, the change in 5 of the 6 parameters is extremely small, $\lesssim 0.4 \%$. But the value of the 6th parameter-the optical depth $\tau$-is increased by $\sim 9.8 \%$ ! We will see that this change then leads to the alleviation of the tension between observations and theoretical predictions based on the $S A$. Note that in spite of this significant change in the value of $\tau$, LQC leaves the highly successful predictions of standard inflation at small angular scales unaffected. In particular, all the finer features of various power spectra predicted by standard inflation for $\ell>30$-where the observational error bars are small-are present also in the LQC prediction. Thus the LQC analysis provides an explicit example supporting a conclusion of Chowdhury et al. (2019) that trans-Planckian effects are not a "threat to inflation".

The paper is organized as follows. Section 2 summarizes the procedure used in observational cosmology to arrive at the 6 parameter $\Lambda \mathrm{CDM}$ model and explains the two anomalies and their significance in greater detail. Section 3 summarizes the basic results from LQC that are used in the subsequent analysis. In particular, we explain the origin of the surprising interplay between the ultraviolet and the infrared that is a rather robust feature of the LQC approaches. The main results are presented in Section 4. They include a discussion of: 1) the LQC corrected primordial power spectrum for scalar perturbations; 2 ) the TT, temperature-electric polarization (TE), the electric polarization $(\mathrm{EE})$, and the lensing potential $(\phi \phi)$ power spectra we predict, and comparisons with those obtained using the $S A$ as well as with the observed power spectra reported by the PLANCK team in their final analysis (Aghanim et al., 2020b). As usual these power spectra are presented in terms of the spherical harmonic components $C_{\ell}$ of the respective correlation functions; 3 ) the TT correlation function $C(\theta)$ predicted by LQC and its comparison with the prediction of the $S A$ as well as PLANCK observations; 4) the $A_{L}$ vs. $\tau$ plots that show that the observed values fall in the $1 \sigma$ contour in LQC, but not if one uses the $S A$; and, 5) the power spectrum for BB polarization predicted by LQC and its comparison with that predicted by the $S A$. The detailed calculations underlying these plots were performed using the Starobinsky and quadratic potentials. The first is preferred phenomenologically while the second has been used often because of its simplicity. Close agreement between the two sets of results is an indication of robustness of the LQC results. In Section 5 we summarize the main results and put them in a broader context.

So far the discrepancy between the results of the SHOES team (Riess et al., 2019) and CMB measurements (Adam et al., 2020) associated with the value of the Hubble parameter has not been systematically addressed in LQC. This is in large part because it is not yet clear whether there is a definitive tension, or if the 
discrepancy is primarily due to systematic calibration offsets (Efstathiou, 2020). Observations may decide on this issue in the near future.

\section{THEORETICAL PREDICTIONS AND PLANCK OBSERVATIONS}

This section is addressed primarily to the LQG community. In Section 2.1 we summarize the procedure used by the PLANCK team to arrive at the six parameter $\Lambda$ CDM model and in Section 2.2 we explain the power suppression and the lensing amplitude anomalies in a bit more detail.

\subsection{The 6 Parameter $\Lambda$ CDM Model}

The six parameters that characterize the $\Lambda \mathrm{CDM}$ Universe can be neatly divided in three groups. The first two parameters-the amplitude $A_{s}$ and the spectral index $n_{s}$ for scalar modes-feature in the $S A$ for the primordial power spectrum:

$$
\mathcal{P}_{\mathcal{R}}(k)=A_{s}\left(\frac{k}{k_{\star}}\right)^{n_{s}-1}
$$

Here $k$ is the wave number in the Fourier decomposition and $k_{\star}$ is a pivot scale (set to $k_{\star}=0.002 \mathrm{Mpc}^{-1}$ in the WMAP analysis and $k_{\star}=0.05 \mathrm{Mpc}^{-1}$ in the PLANCK analysis). If we had $n_{s}=1$, the primordial spectrum would be scale invariant, i.e., it would be independent of the wave number $k$ of the cosmological perturbation. If $n_{s}$ is less than 1 (as observations imply) then there is more power at small $k$, i.e., the power spectrum has a red tilt (One can also consider the possibility of a running $n_{s}$, where it has a $k$ dependence but we will not need this generality.) The second set of parameters, the baryonic and cold matter densities $\Omega_{b} h^{2}$ and $\Omega_{c} h^{2}$, are important for the propagation of cosmological perturbations starting from the end of inflation. The last group of parameters are $100 \theta_{M C}$, that characterizes the angular scale of acoustic oscillations, and the optical depth $\tau$ that characterizes the reionization epoch. These two parameters govern the propagation of perturbations from the last scattering surface to now. Thus, given these six parameters one can use the known astrophysics to propagate the cosmological perturbations starting from the end of inflation, providing us with the theoretical predictions for power spectra we should observe now.

More precisely, for each choice of the six parameters, one can calculate 4 correlation functions $C_{\ell}^{\mathrm{TT}}, C_{\ell}^{\mathrm{TE}}, C_{\ell}^{\mathrm{EE}}, C_{\ell}^{\phi \phi}$ that are the spherical harmonic decompositions of the corresponding correlation function $C^{\mathrm{XY}}(\theta)$ (where $\theta$ characterizes the angular separation of two points in the sky). These correlation functions can be measured and compared with the theoretical predictions, expressed as functions of the six parameters. The statistical analysis, usually done by employing Markov-Chain Monte Carlo method, then leads to the posterior probability distribution for the six parameters. In particular, the maximum likelihood value of the marginalized probability distributions yield the values of six parameters that determine a $\Lambda$ CDM Universe. Observations of the PLANCK collaboration

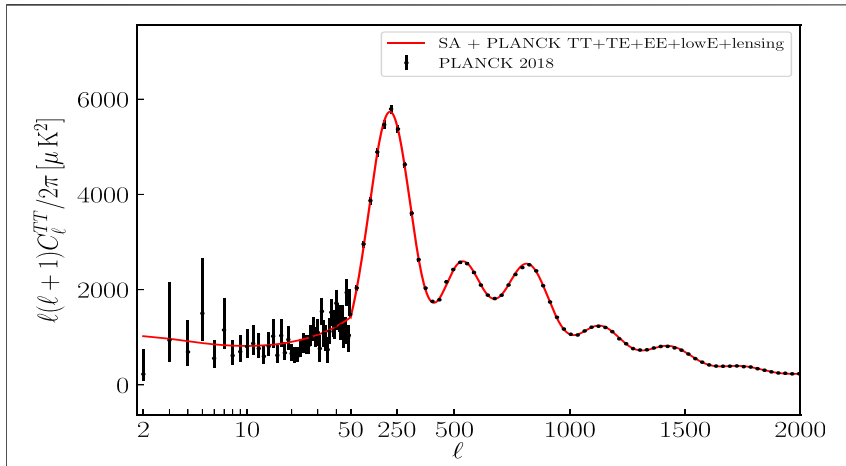

FIGURE 1| The TT-power spectrum. The (red) continuous curve is the theoretical prediction from standard ansatz while the (black) dots with error bars represent the measurements of the PLANCK team reported in 2018.

There is excellent agreement between theory and observations for $\ell>50$ but the observed power is suppressed relative to the theoretical prediction for $\ell \leq 30$. As usual, the horizontal axis uses a logarithmic scale for $\ell \leq 50$ but a linear scale for $\ell \gtrsim 50$.

provide these values (together with the corresponding 1-sigma spreads); this is the "Universe according to PLANCK" (within the $68 \%$ confidence level, characterized by the $1 \sigma$ contours).

Once these parameters are determined, one can calculate additional observable quantities assuming that model and, by carrying out measurements, one can subject the model to consistency tests. For example, the lensing amplitude $A_{L}$ is set to unity in this construction. One can let this parameter vary and test if this value is consistent with observations. Another type of test is provided by the (odd parity) B-modes. In any one model, one can calculate the correlation function $C_{\ell}^{\mathrm{BB}}$. As we discuss in Section 5, several observational missions will soon measure this correlation function with accuracy that may be sufficient to distinguish one model from another (Matsumura et al., 2014; Delabrouille et al., 2018; Hanany et al., 2019). Similarly, the reionization depth $\tau$ will be measured by missions that are unrelated to the CMB (Fialkov and Loeb, 2016). They will constrain $\tau$, providing us with independent checks on the current $\Lambda$ CDM model.

\subsection{Anomalies}

As Figure 1 shows, the TT power spectrum is in excellent agreement with the theoretical predictions using the $S A$ at small angular scales $(\ell>50)$. This is especially noteworthy because the instrumental errors are truly minuscule in this range.

However, for $\ell \leqq 30$ the observed power is lower than the theoretical prediction. This power suppression was evident already in the WMAP data, and is reenforced by the PLANCK findings. Over the years it has been argued (Spergel et al., 2003; Sarkar et al., 2011; Akrami et al., 2019; Schwarz et al., 2016) that this anomaly is brought to forefront if one carries out the comparison using the quantity $S_{1 / 2}:=\int_{-1}^{1 / 2}[C(\theta)] \mathrm{d}(\cos \theta)$ that features the physical space TT correlation function $C(\theta)$ in place of the spherical harmonic coefficients $C_{\ell}$. Qualitatively, large angular scales correspond to small $\ell$ in the spherical harmonic decomposition. However, for any given $\theta_{o}$ the value $C\left(\theta_{o}\right)$ 

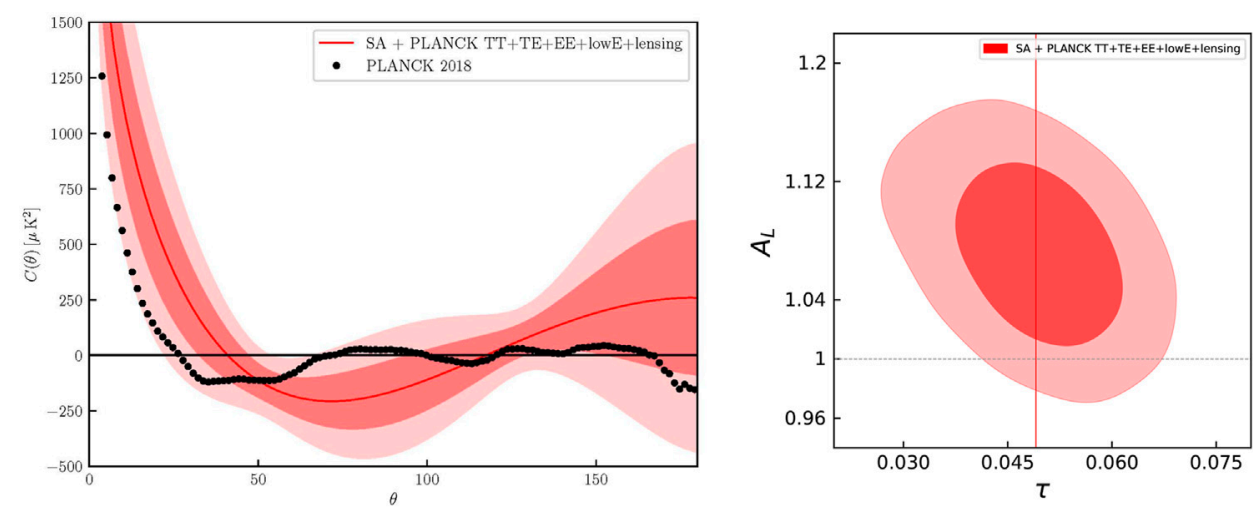

FIGURE 2 | Left Panel: Large scale power anomaly as measured by $C(\theta)$. The (red) continuous curve is the theoretical prediction from the $S A$ with 68 and $95 \%$ confidence level contours arising from cosmic variance, while the (black) dots represent the 2018 PLANCK team measurements. The $S A$ prediction for $S_{1 / 2}$ is more than 35 times the observed value. Right Panel: Lensing amplitude $A_{L}$ vs. optical depth $\tau A_{L}=1$ lies outside the $1 \sigma$ contour, signaling the tension between theory and observations.

receives contributions from all $\ell$. Therefore $S_{1 / 2}$ is a more direct measure of the cumulative power for $\theta \geq 60^{\circ}$ than the $C_{\ell}$ 's for low $\ell$ s. Indeed, as the left panel in Figure 2 vividly shows, the observed $C(\theta)$ (the black, dotted curve) is very close to zero for $\theta>60^{\circ}$, in contrast to the theoretical (solid, red) curve. The observed value of $S_{1 / 2}$ is $\sim 1209$, while the theoretical prediction from the $S A$ is $42,496.5$, some 35 times larger. Since the extent of this discrepancy is not immediate from Figure 1, one may wonder why the power spectrum is not reported using $C^{\mathrm{TT}}(\theta)$ in place of $C_{\ell}^{\mathrm{TT}}$. The reason is that the $C_{\ell}^{\mathrm{TT}}$ for distinct $\ell$ s are (almost) uncorrelated and can therefore be treated as independent observables, while $C^{\mathrm{TT}}(\theta)$ for distinct $\theta$ have massive cross-covariance, whence the statistical significance of power suppression is only $2-3 \sigma$ in spite of the large deviation seen in the left panel of Figure 2. Also, because of these correlations, to obtain the 1 and $2 \sigma$ contours in this plot one has to take into account a large covariance matrix which in turn requires a detailed understanding of the instruments and the masking procedure near $\theta=180^{\circ}$ used in the data analysis to remove the contamination coming from the galactic plane.

The second anomaly is associated with the lensing amplitude $A_{L}$ depicted in the right panel of Figure 2 . As it propagates from the last scattering surface at $z \approx 1100$ to us, the $\mathrm{CMB}$ is lensed due to inhomogeneities. The lensing potential is nearly Gaussian because there are many lenses along the line of sight. As explained in Section 2.1, the six parameter $\Lambda \mathrm{CDM}$ Universe is determined using best fits to all four power spectra. Once this is done, one can compare each observed power spectrum, one by one, with the theoretically predicted power spectrum for that specific $\Lambda \mathrm{CDM}$ Universe. Just as this comparison revealed an anomalous suppression of power in $C_{\ell}^{\mathrm{TT}}$ for $\ell<30$, one finds an anomaly also in the lensing potential power spectrum $C_{\ell}^{\phi \phi}$ : Relative to the prediction of the best-fit $\Lambda \mathrm{CDM}$ model, there is power enhancement in the range $8 \leq \ell \leq 400$ used by the PLANCK collaboration to report the baseline cosmological results (Adam et al., 2020) (In this range, the reconstruction procedure is robust and the impact of systematics is reduced). As a consistency check on the 6-parameter $\Lambda \mathrm{CDM}$ model, one introduces a 7 th phenomenological parameter $A_{\mathrm{L}}$-the lensing amplitude, normalized so that $A_{\mathrm{L}}=1$ in the 6 parameter $\Lambda \mathrm{CDM}$ model-and allows it to vary. Varying $A_{\mathrm{L}}$ can be considered as a conservative way of marginalizing over the systematics of the PLANCK data. Departure of $A_{\mathrm{L}}$ from unity signals a tension with predictions based on the standard 6-parameter $\Lambda \mathrm{CDM}$. One finds that $A_{\mathrm{L}}$ is higher than 1 at $\sim 1.9 \sigma$ level (Adam et al., 2020). This is the lensing amplitude anomaly and its occurrence has been interpreted as a hint of new physics (Di Valentino and Bridle, 2018). The right panel of Figure 2 illustrates this tension. Here $A_{\mathrm{L}}$ is plotted against the optical depth $\tau$ because this plot will be useful when we compare the results from LQC with those from the SA in Section 4: Of the six parameters, only $\tau$ receives significant corrections from LQC. $\tau$ is singled out even within the $S A$ by the fact that the relative error (as measured by the ratio of the standard deviation to the mean value) in $\tau$ is $\sim 13 \%$ while that in the other five $\Lambda \mathrm{CDM}$ parameters are less than $1 \%$. In the plot, the tension is manifested in the fact that the line $A_{\mathrm{L}}=1$ lies outside the $1 \sigma$ contour. Attempts to alleviate this tension within the standard paradigm based on general relativity (GR)-e.g. changing the background geometry by introducing spatial curvature-are not supported by lensing reconstruction or Baryonic oscillations (BAO) data (since the joint constraint with $\mathrm{BAO}$ is consistent with flat Universe, with $\Omega_{\mathrm{K}}=0.001 \pm 0.002$ ).

As noted in the introduction, while the statistical significance of either of these anomalies is low, together the two imply that the observed Universe will emerge only once in $\sim 10^{6}$ realizations of the posterior probability distributions. Therefore, as the PLANCK collaboration suggested both in its 2015 and 2018 data releases, alleviation of this tension is of considerable interest especially if the mechanism is rooted in physics beyond GR (Ade et al., 2016; Aghanim et al., 2020a).

\section{LOOP QUANTUM COSMOLOGY}

This section is addressed primarily to the cosmology community. In Section 3.1 we briefly recall how quantum geometry effects 
underlying LQG lead to a resolution of the big bang singularity, replacing it with a big bounce. Since physical quantities do not diverge anywhere, one can extend the standard inflationary scenario all the way to the bounce. In Section 3.2, we discuss the pre-inflationary dynamics of cosmological perturbations, specifically the propagation of quantum fields representing these perturbations on the quantum background geometry provided by LQC. In Section 3.3, we explain why, contrary to one's initial expectations, this pre-inflationary dynamics can leave observable signatures at large scales in the CMB. We will use this framework in Section 4 to extract the LQC corrections to the primordial power spectrum. As mentioned in Section 1, together with observations, these corrections imply that we live in a somewhat different $\Lambda \mathrm{CDM}$ Universe in which the two anomalies are naturally alleviated.

\subsection{The Big Bounce of LQC}

Investigations of the early Universe are often carried out assuming that spacetime is well approximated by a spatially flat FLRW background metric of GR, together with first order cosmological perturbations that are described by quantum fields. Consider the inflationary paradigm and, for brevity, let us refer to the time when the pivot mode $k=k \star$ exits the Hubble horizon simply as 'the onset of inflation'. At this onset, while spacetime curvature is huge by astrophysical standards-some $10^{65}$ times that at the horizon of a solar mass black hole-it is only $\sim 10^{-12}$ times the Planck scale. Therefore, at the level of accuracy of current interest, it is safe to ignore the quantum gravity effects even at the onset of inflation. Since spacetime geometry is well approximated by a (perturbed) de Sitter metric at this time, one assumes that the quantum fields representing cosmological perturbations are in the Bunch-Davies (BD) vacuum that is selected by the isometries of the de Sitter metric and evolves the perturbations to the future (as curvature decreases further).

However, conceptually it is rather ad-hoc to begin, so to say, 'in the middle' of evolution. If we go further back in the past, curvature attains the Planck scale, and then diverges at the big bang. During this pre-inflationary epoch, spacetime geometry is not at all well-approximated by the de Sitter geometry. Why, then, can we assume the state to be the $\mathrm{BD}$ at the onset of inflation? Should we not start in the deep Planck regime and check whether the state is in fact in the BD vacuum at this onset? This would require quantum cosmology, where the Friedmann, Lemaitre, Robertson, Walker (FLRW) solution of Einstein's equations, characterized by the scale factor $a(t)$ and a matter field $\phi(t)$, is replaced by a quantum state $\Psi(a, \phi)$ subject to an appropriate quantum version of Einstein-matter field equations. Note that reference to the proper time $t$ has disappeared-quantum dynamics is relational, à la Leibnitz: for example, one can use the matter field $\phi$ as an internal clock, and describe how the scale factor evolves with respect to it. Quantum fields representing cosmological perturbations are now to propagate on a quantum FLRW geometry $\Psi(a, \phi)$ which assigns probability amplitudes to various metrics, rather than on a single FLRW spacetime.

While this general viewpoint is common to all quantum cosmologies, LQC has two key features that distinguish it from the older Wheeler-DeWitt (WDW) theory, often called quantum geometrodynamics. First, as explained below, the mathematical framework of LQC descends from the welldeveloped kinematics of LQG, using a symmetry reduction tailored to homogeneity and isotropy. In the WDW theory one is yet to develop rigorous kinematics for full quantum geometrodynamics; because issues related to the presence of an infinite number of degrees of freedom are generally ignored, the underlying mathematical framework has remained formal. In quantum cosmology, then, one introduces structures like the WDW equation without guidance from a more complete framework. This leads to the second key difference. The LQC quantum Einstein's equation is qualitatively different from the WDW equation, in that it mirrors features of the quantum Riemannian geometry of full LQG. As a direct result, strong cosmological singularities-and in particular the big bang-are naturally resolved in LQG (Singh, 2009; Ashtekar and Singh, 2011; Agullo and Singh, 2017).

We will now explain these differences in some detail. As is common in quantum field theories, in full LQG one begins with the Heisenberg algebra $\mathcal{A}$ of basic ('canonically conjugate') observables, called the holonomy-flux algebra (Ashtekar and Isham, 1992; Ashtekar and Lewandowski, 2004; Giesel, 2017). We then have a highly non-trivial result that ensures that $\mathcal{A}$ admits a unique representation by operators of a Hilbert space $\mathcal{H}$ that respects the 'background independence' or 'diffeomorphism covariance' of the theory (Lewandowski et al., 2006; Fleishchack, 2009). This representation underlies the rigorous kinematical framework of LQG. In particular, one finds that geometrical observables are well-defined self-adjoint operators with discrete eigenvalues. Of particular interest is the area gap-the first nonzero eigenvalue $\Delta$ of the area operator. It is a fundamental microscopic parameter of the theory that then governs important macroscopic phenomena in LQC that lead, e.g., to finite upper bounds for curvature. ${ }^{1}$ In LQC, one first reduces the holonomy-flux algebra $\mathcal{A}$ used in full LQG to a smaller symmetry reduced algebra $\mathcal{A}_{\text {red }}$. Again there is a uniqueness theorem that guarantees that $\mathcal{A}_{\text {red }}$ admits a unique representation on a Hilbert space $\mathcal{H}_{\mathrm{LQC}}$ that respects the action of the (residual) diffeomorphism group on $\mathcal{A}_{\text {red }}$ (Ashtekar and Campiglia, 2012; Engle et al., 2017). This representation is qualitatively different (i.e. unitarily inequivalent) from the Schrödinger representation used in the WDW theory. In particular, the differential operator representing the gravitational part in the WDW equation is not even defined on $\mathcal{H}_{\mathrm{LQC}}$; it is naturally replaced by a certain difference operator that explicitly involves the area gap $\Delta$ (Ashtekar et al., 2006a; Ashtekar et al., 2006b; Ashtekar and Singh, 2011). One can now start with a quantum state $\Psi(a, \phi)$ that is peaked on the classical dynamical trajectory at a suitably late time when curvature is low, and evolve it back in time toward the big bang using either the WDW equation or the LQC evolution equation. Interestingly the wave function

${ }^{1}$ This is because the curvature operator is defined by considering 'Aharanov-Bohm fluxes' across small surfaces $S$ and then shrinking the surface till it has the minimum area $\Delta$. 
continues to remain sharply peaked in both cases. In the WDW theory it follows the classical trajectory all the way into the singularity, while in LQC it ceases to follow the classical trajectory once the curvature is about $\sim 10^{-4}$ times the Planck curvature. Then the quantum geometry corrections dominate and the wave function $\Psi(\phi, a)$ bounces when the curvature and matter density attain their upper bounds. In this backward evolution, curvature starts decreasing after the bounce and the Universe expands. Once the curvature falls below $\sim 10^{-4}$ times the Planck curvature, the wave function again follows a classical trajectory which is now expanding in the past direction [For details, see Ashtekar et al. (2006b), Ashtekar et al. (2008), Ashtekar and Singh (2011), Agullo and Singh (2017)].

Thus, key differences between LQC and the WDW theory arise from the fact that the WDW theory has no knowledge of the quantum nature of Riemannian geometry that LQC inherits from LQG. Indeed, there is a precise sense in which the LQC evolution equation reduces to the WDW differential equation in the limit in which the area gap goes to zero (Ashtekar et al., 2008). The upper bound of curvature in LQC is given by $\operatorname{curv}_{\max }=\left[3\left(24 \pi^{2}\right) /\left(2 \Delta^{3}\right)\right] \ell_{\mathrm{Pl}}^{4} \simeq 62 \ell_{\mathrm{Pl}}^{-2}$, where, in the last step, we have used the numerical value $\tilde{\Delta} \simeq 5.17 \ell_{\mathrm{Pl}}^{2}$ of the area gap. In any LQC solution $\Psi_{\text {lqc }}(a, \phi)$, the curvature attains its maximum value at the bounce and this value is extremely well approximated by curv $_{\max }$ if the state is sharply peaked. Note that the upper bound diverges as $\Delta \rightarrow 0$, in line with the finding that curvature grows unboundedly as one evolves the WDW state $\Psi_{\mathrm{wdw}}(a, \phi)$ to the past. By contrast, in LQC, while the quantum geometry effects are negligible away from the Planck regime, they become dominant in the Planck regime, creating an effective repulsive force of quantum origin that causes the Universe to bounce.

It is interesting that this force rises and falls extremely rapidly, making the agreement with GR excellent outside the Planck regime. However, it has a very non-trivial global effect, in that physics does not stop at the big bang as in GR. Rather, there is an expanding FLRW Universe to the future of the bounce and a contracting FLRW Universe to the past, joined by a 'quantum bridge'. These qualitatively new features arise without having to introduce matter that violates any of the standard energy conditions, and without having to introduce new boundary conditions, such as the Hartle-Hawking 'no-boundary proposal'; they are consequences just of the quantum corrected Einstein's equations. Thus, the existence of the bounce and the upper bound on curvature and matter density can be directly traced back to quintessential features of quantum geometry. These considerations have been extended beyond the spatially flat FLRW models to include spatial curvature, non-zero cosmological constant, anisotropies [see, e.g. Ashtekar and Singh (2011), Agullo and Singh (2017), and references therein] as well as the simplest inhomogeneities captured by the Gowdy models in GR, and also to the Brans-Dicke theory [see, e.g. Zhang et al. (2013), Elizaga Navascués et al. (2015)]. Taken together, these results bring out the robustness of the LQC bounce.

Since the area gap plays an important role in the LQC dynamics, before concluding this subsection, we will make a small detour to explain how its numerical value $\tilde{\Delta} \simeq 5.17 \ell_{\mathrm{Pl}}^{2}$ is arrived at. Recall, first, that in QCD there is a quantization ambiguity-parametrized by an angle $\theta$-because of the freedom in adding a topological term to the action. One encounters a similar quantization ambiguity in LQG (again associated with the freedom to add a term to the action that does not affect equations of motion), encoded in the so-called Barbero-Immirzi parameter, $\gamma>0$, which trickles down to the expressions of observables on $\mathcal{H}$, such as the area operator $\widehat{A}_{S}$. The eigenvalues of $\widehat{A}_{S}$ are discrete in all $\gamma$-sectors. But their numerical values are proportional to $\gamma$ and vary from one $\gamma$ sector to another. Observables also have a $\theta$ dependence in QCD and the value of $\theta$ that Nature has selected is determined experimentally. In LQG, a direct measurement of eigenvalues of geometric operators would determine $\gamma$. But of course such a measurement is far beyond the current technological limits. However one can use thought experiments. Specifically, in LQG the number of microstates of a black hole horizon grows exponentially with the area, whence one knows that the entropy is proportional to the horizon area (Ashtekar et al., 1998; Ashtekar et al., 2000). But the proportionality factor depends on the value of $\gamma$. Therefore if one requires that the leading term in the statistical mechanical entropy of a spherical black hole should be given by the Bekenstein-Hawking formula $S=A / 4 \ell_{\mathrm{Pl}}^{2}$, one determines $\gamma$ and thus the LQG sector Nature prefers. In this sector the explicit value of the area gap yields $\Delta \simeq 5.17 \ell_{\mathrm{Pl}}^{2}$ (Domagala and Lewandowski, 2004; Meissner, 2004; Barbero and Perez, 2017; Perez, 2017) (and the leading term in the entropy of more general black holes-not necessarily spherical-agrees with the Bekenstein-Hawking formula). This is the value used in LQC calculations. $^{2}$

This concludes our broad-brush overview of how quantum geometry considerations lead to a natural resolution of the big bang singularity in LQC. The resolution has been analyzed in detail in a large number of LQC papers, using Hamiltonian, cosmological-spinfoam and 'consistent histories' frameworks [see, e.g. Ashtekar et al. (2006b), Ashtekar et al. (2009a), Ashtekar et al. (2010), Ashtekar and Singh (2011), Craig and Singh (2013), Agullo and Singh (2017)].

Remark: Recently some concerns have been expressed about the simplicity of the LQC description of the early Universe, and on whether "general physics principles of effective field theory and covariance" have been appropriately incorporated (Bojowald, 2020). Many of the specific technical points were already addressed, e.g., in Ashtekar and Singh (2011), Corichi and Singh (2008), Kaminski and Pawlowski (2010) and in the Appendix of Ashtekar (2009). In addition, we would like to clarify possible confusion on the following points. First, although 'effective equations' are often used in LQC, conceptually they are on a very different footing from those used in effective field theories: One does not integrate out the UV

\footnotetext{
${ }^{2}$ There are two closely related but technically different ways of characterizing the quantum states of an isolated horizon representing a black hole in equilibrium (Barbero and Perez, 2017). They lead to slightly different values of the Barbero-Immirzi parameter $(0.237$ and 0.274$)$ and hence of the area gap $\left(5.17 \ell_{\mathrm{Pl}}^{2}\right.$ and $\left.5.98 \ell_{\mathrm{Pl}}^{2}\right)$. Because the values are very close, our results are not sensitive to these differences. See Section 4.3 .
} 
modes of cosmological perturbations. The term 'effective' is used in a different sense in LQC: these equations carry some of the leading-order information contained in sharply peaked quantum FLRW geometries $\Psi(a, \phi)$. As we will see in Section 3.2, equations satisfied by the cosmological perturbations are indeed covariant. On the issue of simplicity of the LQC description, we note that in the 1980s it was often assumed that the early Universe is irregular at all scales and therefore quite far from being as simple as is currently assumed at the onset of inflation. Yet now observations support the premise that the early Universe is exceedingly simple in that it is well modeled by a FLRW spacetime with first order cosmological perturbations (Chowdhury et al., 2019). Therefore, although a priori one can envisage very complicated quantum geometries, it is far from being clear that they are in fact realized in the Planck regime. Nonetheless, one should keep in mind that, as in other approaches to quantum cosmology, in LQC the starting point is the symmetry reduced, cosmological sector of GR. Difference from the Wheeler-DeWitt theory is that one follows the same systematic procedure in this sector as one does in full LQG. But the much more difficult and fundamental issue of systematically deriving LQC from full LQG is still open mainly because dynamics of full LQG itself is still a subject of active investigation. See, e.g., Assanioussi et al. (2018), Olmedo and Alesci (2019) as illustrations of the current status.

\subsection{Cosmological Perturbations in the Pre-inflationary Era of LQC}

In inflationary paradigms the Mukhanov-Sasaki scalar modes ${ }^{3}$ of cosmological perturbations are represented by quantum fields $\widehat{\mathcal{Q}}$ that propagate on a background FLRW metric $g_{a b}$. The use of a classical background geometry is justified since, as explained above, spacetime curvature is twelve orders of magnitude below the Planck scale even at the onset of inflation. However, to extend the paradigm all the way to the LQC bounce, one has to replace the metric $g_{a b}$ of GR with an LQC wave function $\Psi(a, \phi)$ because assumptions underlying quantum field theory (QFT) on curved spacetimes fail in the Planck regime. At first the task seems daunting: How do you evolve quantum fields when you have only a probability distribution $\Psi(a, \phi)$ for various spacetime geometries rather than a single metric $g_{a b}$ ? Fortunately, there is an unexpected simplification (Ashtekar et al., 2009b; Agullo et al., 2012; Agullo et al., 2013b): So long as $\Psi(a, \phi)$ is sharply peaked, and the back reaction of the perturbations $\widehat{\mathcal{Q}}$ on the background quantum geometry $\Psi$ remains negligible, dynamics of quantum fields $\widehat{\mathcal{Q}}$ on $\Psi$ is extremely well-approximated by that of quantum fields $\widehat{\mathcal{Q}}$ propagating on a smooth, quantum corrected FLRW metric $\tilde{g}_{a b}$ which is constructed in a precise manner from $\Psi$. As one would expect, coefficients of $\tilde{g}_{a b}$ depend on $\hbar$. In the literature, $\tilde{g}_{a b}$ is often called the dressed metric. It is

${ }^{3}$ In the pre-inflationary epoch, the curvature perturbation $\widehat{\mathcal{R}}$ for scalar modes become ill-defined at the turn-around point where $\dot{\phi}=0$. Therefore, in the LQC literature, one uses the Mukhanov-Sasaki gauge invariant scalar perturbation $\widehat{Q}$ in the pre-inflationary dynamics and converts the result to $\widehat{\mathcal{R}}$ at the end of inflation. 'dressed' by certain quantum fluctuations in $\Psi(a, \phi)$ specified below; it carries the information in the quantum geometry $\Psi(a, \phi)$ that the propagation of cosmological perturbations is sensitive to.

The construction of the dressed metric $\tilde{g}_{a b}$ can be summarized as follows. Recall first that in the standard inflationary scenario, the Mukhanov-Sasaki quantum field $\widehat{\mathcal{Q}}$ satisfies a wave equation $\left(\square+\mathcal{U} / a^{2}\right) \widehat{\mathcal{Q}}=0$ where $\square$ is the d'Alembertian w.r.t. to the background FLRW metric $g_{a b}$ (satisfying the unperturbed, zeroth order Einstein's equations) and $\mathcal{U}$ is constructed from the inflationary potential and the background FLRW solution [see, e.g. Agullo et al. (2013b)]. At the classical level, this evolution equation can be derived starting with the full Hamiltonian constraint of GR coupled with the scalar field, and then appropriately truncating it to second order in perturbations (Agullo et al., 2013a). In LQC, the background quantum geometry $\Psi(a, \phi)$ satisfies the zeroth-order LQC Hamiltonian constraint. The scalar mode $\widehat{\mathcal{Q}}$ propagates on this $\Psi(a, \phi)$ and its dynamics is governed by the appropriate second order truncation of the full Hamiltonian constraint. If the state $\Psi(a, \phi)$ is sharply peaked and the back reaction of the perturbation $\widehat{\mathcal{Q}}$ is negligible, then one has the following result (Ashtekar et al., 2009b): Propagation of $\widehat{\mathcal{Q}}$ on the quantum geometry $\Psi(a, \phi)$ is very well approximated by that of a quantum field $\widehat{\mathcal{Q}}$ satisfying $\left(\tilde{\square}+\tilde{\mathcal{U}} / \tilde{a}^{2}\right) \widehat{\mathcal{Q}}=0$. Here $\tilde{\square}$ is the d'Alembertian with respect to the dressed metric

$$
\tilde{g}_{a b} \mathrm{~d} x^{a} \mathrm{~d} x^{b} \equiv \mathrm{d} \tilde{s}^{2}=\tilde{a}^{2}\left(-\mathrm{d} \tilde{\eta}^{2}+\mathrm{d} \vec{x}^{2}\right)
$$

with

$$
\frac{\tilde{a}^{4}=\left\langle\widehat{H}^{-\frac{1}{2}} \widehat{a}^{4}(\phi) \widehat{H}^{-\frac{1}{2}}\right\rangle}{\left\langle\hat{H}^{-1}\right\rangle \text { and } \mathrm{d} \tilde{\eta}=\left\langle\widehat{H}^{-1 / 2}\right\rangle\left(\left\langle\widehat{H}^{-1 / 2} \widehat{a}^{4}(\phi) \hat{H}^{-1 / 2}\right\rangle\right)^{1 / 2} \mathrm{~d} \phi}
$$

and $\tilde{\mathcal{U}}(\phi)$ is the dressed effective potential

$$
\frac{\tilde{\mathcal{U}}(\phi)=\left\langle\widehat{H}^{-\frac{1}{2}} \widehat{a}^{2}(\phi) \hat{\mathcal{U}}(\phi) \widehat{a}^{2}(\phi) \widehat{H}^{-\frac{1}{2}}\right\rangle}{\left\langle\hat{H}^{-\frac{1}{2}} \widehat{a}^{4}(\phi) \hat{H}^{-\frac{1}{2}}\right\rangle}
$$

All operators and their expectation values refer to the Hilbert space of the background FLRW quantum geometry: the expectation values are taken in the state $\Psi, \widehat{H}$ is the 'free' Hamiltonian in absence of the inflaton potential, and $\hat{a}(\phi)$ is the (Heisenberg) scale factor operator (Ashtekar et al., 2009b; Agullo et al., 2013b).

At first, the result seems surprising. But physically it can be understood using a simple analogy with propagation of light in a medium such as water. In the full quantum description, individual photons interact with the molecules of the material. However, the key features of propagation can be extracted simply by computing a few macroscopic parameters such as the refractive index and birefringence that can be extracted from the microstructure of the material. Other details of the quantum state of the medium are not important to study propagation. In this analogy, the cosmological perturbation plays the role of light and quantum geometry, the role of the medium. To determine the propagation of $\widehat{\mathcal{Q}}$, one needs to extract only $\tilde{a}, \tilde{\eta}$ and $\tilde{\mathcal{U}}$ from the 
quantum state $\Psi(a, \phi)$. The rest of the very rich information of quantum geometry it contains is not directly relevant (Incidentally, the tensor modes satisfy the wave equation for the same dressed metric $\tilde{g}_{a b}$; as in standard inflation, there is no dependence on the potential.)

It is clear from the form of Eqs 3.2, 3.3 that the expressions of the dressed metric and the dressed potential could not have been guessed a priori. They resulted from explicit, detailed calculations (Ashtekar et al., 2009b; Agullo et al., 2013b). The observable predictions reported in Section $\mathbf{4}$ are obtained by first calculating the dressed metric and the dressed potential starting from the given quantum geometry $\Psi(a, \phi)$ and then evolving the scalar mode using $\left(\tilde{\square}+\tilde{\mathcal{U}} / \tilde{a}^{2}\right) \widehat{Q}=0$.

Remarks

1. For clarity, let us spell out the conceptual elements of the procedure used to extract dynamics of cosmological perturbations since there is occasional confusion on this point. The starting point in LQC is the Hamiltonian formulation of GR coupled to the inflaton (albeit in the connection variables used in LQG). One then extracts the sector of full GR that corresponds to the homogeneous isotropic fields (which serve as the background) together with first order perturbations. It is this classical theory that is then quantized using LQG techniques (Agullo et al., 2013a). Dynamics of quantum perturbations are governed by the Hamiltonian constraint operator of the truncated sector, where both the background geometry and perturbations are treated quantum mechanically. One does not simply assume that perturbations satisfy linearized equations of GR on a bouncing classical metric. That the dynamics of perturbations is well approximated by quantum field satisfying an evolution equation involving $\tilde{g}_{a b}$ and $\tilde{\mathcal{U}}$ is a result that holds under conditions spelled out above. Note also that the equation is covariant w.r.t. $\tilde{g}_{a b}$ and $\tilde{g}_{a b}$ rapidly tends to the classical FLRW metric of GR outside the Planck regime.

2. Initially, analysis of Ashtekar et al. (2009b) suggested that the propagation of $\widehat{\mathcal{Q}}$ on the quantum geometry $\Psi(a, \phi)$ would be exactly the same as that on the corresponding dressed metric 3.1 and potential (3.3) for any $\Psi(a, \phi)$. However, Kaminski later found (Kamiński, 2012) that there is a subtle infrared problem (that can be missed in numerical simulations since they have to use an infrared cutoff). Kamiński et al. (2020) then showed that, as a result, the implications of Ashtekar et al. (2009b) are not as general; the result would not hold without restrictions on the background quantum geometry $\Psi(a, \phi)$. This situation is qualitatively similar to that, e.g., in quantum electrodynamics which also faces infrared issues in rigorous treatments. However, in QED the ensuing difficulties can be avoided by focusing just on those quantities that are 'infrared safe'. One can adopt a similar strategy in LQC by introducing suitable infrared safe observables through regularization. Furthermore, for states $\Psi(a, \phi)$ that are sufficiently sharply peaked, the regularization ambiguity is completely negligible. Previous calculations of power spectra in LQC [e.g. Agullo et al. (2012), Agullo et al. (2013b), Agullo and Morris (2015), Agullo (2015), Sreenath et al. (2019), Agullo et al. (2021b)], as well as the current investigation, use states that are sufficiently sharply peaked in this sense, whence the use of dressed metric is justified in spite of the infrared difficulties.

\subsection{Primordial Spectrum: Why Pre-inflationary Dynamics Matters}

A natural question now is whether the pre-inflationary phase of LQC dynamics described in the last two subsections has any observable consequences. Let us therefore focus on the observable modes $\widehat{\mathcal{Q}}_{k}$. These have co-moving wavenumbers $k$ in the range $\sim\left(0.1 k_{\star}, 300 k_{\star}\right)$, where $k_{\star}=0.002 \mathrm{Mpc}^{-1}$ is the WMAP pivot scale. The evolution equation for these modes implies that, they 'experience' curvature in the background metric $\tilde{g}_{a b}$ only if their physical wavelength $\lambda(t)=a(t) / k$ is comparable or larger than the radius of curvature $r_{\text {curv }}(t)=(6 / R)^{\frac{1}{2}}$ of $\tilde{g}_{a b}$ corresponding to the scalar curvature $R$ at that time. Let us denote by $t=t_{\star}$ the time at which the relevant slow roll phase starts; this is our onset of inflation. Therefore, a few e-folds before and after $t_{\star}$, the observable modes propagate as though they are in flat spacetime and therefore do not get excited by the background geometry. What happens in the distant past? The left panel of Figure 3 shows the evolution of $r_{\text {curv }}$ (blue solid curve) and of $\lambda$ of observable modes (the gray shaded band), both in GR. Note that in the pre-inflationary epoch $r_{\text {curv }}$ is far from being constant whence the spacetime metric is very different from the de Sitter metric. Since the scalar curvature $R$ diverges at the big bang, $r_{\text {curv }}$ goes to zero. Because the scale factor $a$ of the classical FLRW metric goes to zero, physical wavelengths $\lambda$ also goes to zero at the big bang. However, they do not go to zero as fast as $r_{\text {curv }}$. Therefore, as one approaches the big bang in the past evolution, all observable modes exit the curvature radius, 'experience' curvature at sufficiently early times and get excited. These excitations have to be delicately fine-tuned for the state to be in the $\mathrm{BD}$ vacuum later on, at $t=t_{\star}$ i.e. at the onset of inflation. Put differently, the Heisenberg state representing the $\mathrm{BD}$ vacuum at the onset of inflation is an unnatural choice from the perspective of the Planck regime because it carries certain delicately choreographed excitations there. Of course, one can argue that the quantum field theory in curved spacetime cannot be extrapolated to the Planck regime. But by itself this argument does not provide a justification for using the $\mathrm{BD}$ state at $t=t_{\star}$ either.

In LQC, the situation is quite different. Because the scalar curvature $\tilde{R}$ of the dressed metric $\tilde{g}$ has a finite upper bound $\tilde{R}_{\text {max }} \simeq 62 \ell_{\mathrm{Pl}}^{-2}$, reached at the bounce, $r_{\text {curv }}$ reaches its minimum value $r_{\text {curv }}^{\min } \simeq 0.31 \ell_{\mathrm{Pl}}$, whence it is only those modes which satisfy $\lambda \gtrsim r_{\text {curv }}^{\min }$ at the bounce that experience curvature in their evolution from the bounce to the onset of inflation. In our approach (as discussed below) the background quantum geometry $\Psi(a, \phi)$ is such that only the longest wavelength observable modes satisfy this inequality. This feature is shown in the right panel of Figure 3. Therefore, all but the longest wavelength observable modes propagate from the bounce-time to the onset of inflation as though they are in flat spacetime and hence it is natural that they be in the $\mathrm{BD}$ vacuum at $t=t_{\star}$. It is only the longest wavelength modes that will be excited and hence not in the 

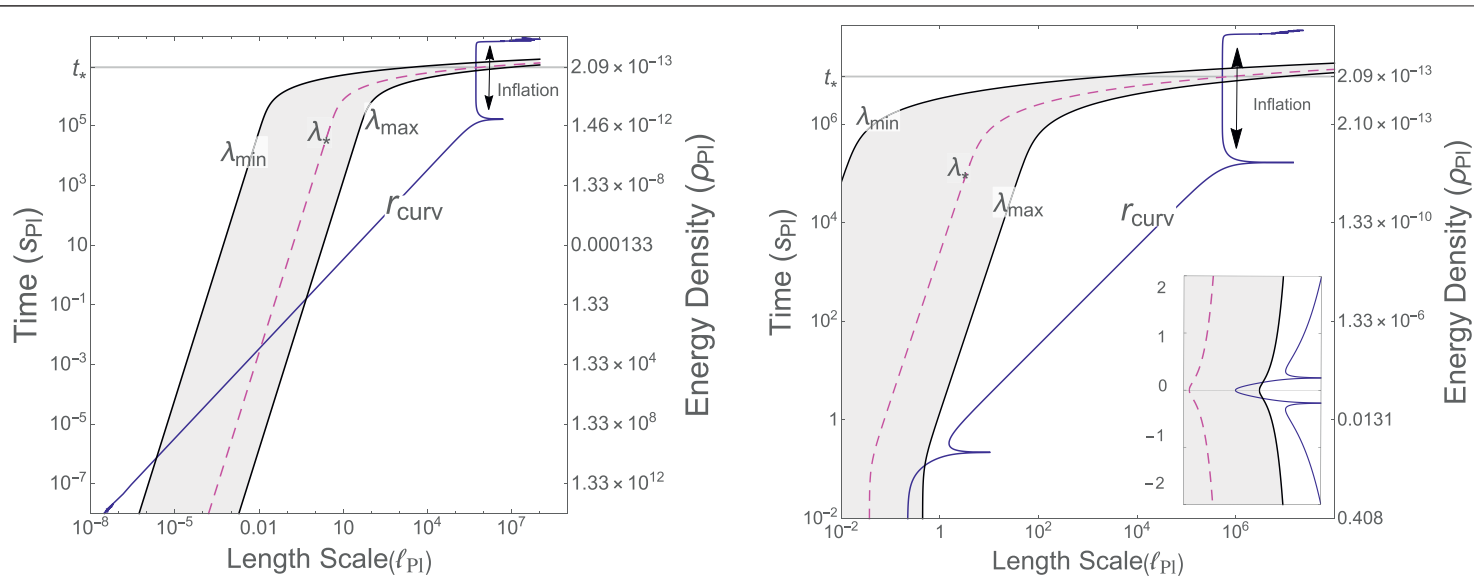

FIGURE 3 | Time dependence of the physical wavelengths $\lambda=a / k$ of modes and radius of curvature $r_{\text {curv }}$ in the pre-inflationary era, using Starobinsky potential. The left vertical axis shows cosmic time $t$ (in Planck seconds) and right vertical axis shows the energy density (also in Planck units). The shaded bands represent the wavelengths of observable modes and the dashed line denotes the WMAP pivot mode with $\lambda_{\star}=a(t) / k_{\star}$. The solid (blue) lines represent the evolution of $r_{\text {curv }}$. Left panel: General Relativity. In the Planck regime near singularity $(t=0)$, all observable modes exit the curvature radius and are thus excited. $\boldsymbol{R}$ ight panel: $L Q C$. Only the longest wavelength modes in the observable band get excited and fail to be in the BD vacuum at $t=t \star$. The inset shows dynamics near the bounce. Plots of the quadratic potential are very similar.

BD vacuum. But won't these excitations get just washed away during inflation? The answer is in the negative: because of spontaneous emission, the number density of these excitations remains constant (Agullo and Parker, 2011a; Agullo and Parker, 2011b; Ganc and Komatsu, 2012). As a result, as we will see in Section 4, the primordial spectrum does differ from that based on the $S A$, but only at largest angular scales.

Note that there is a deep interplay between the UV and IR in LQC. As we saw in Section 2.1, in LQC it is the UV modifications of GR in the Planck regime that tame the big bang singularity and make all physical quantities finite. As a result we have a finite $\tilde{R}_{\max }$ and a non-zero $\tilde{r}_{\text {curv }}^{\mathrm{min}}$. It is then natural for all but the longest wavelength observable modes to be in the $\mathrm{BD}$ vacuum at the onset of inflation. While the LQC corrections to the background geometry are significant in the UV, their effect on cosmological perturbations is non-negligible only in the IR. This is the point that was highlighted in the abstract and Section 1.

Finally, to obtain specific predictions, one needs a quantum state of geometry $\Psi(a, \phi)$ and a quantum state $\psi(\mathcal{Q}, \phi)$ of scalar modes. At this point, different approaches within LQC make different choices [see, e.g. Agullo et al. (2013b), FernandezMendez et al. (2012), Barrau et al. (2014), Ashtekar and Barrau (2015), Agullo and Morris (2015), Agullo (2015), Gomar et al. (2017), Agullo et al. (2018), Barrau et al. (2018), Agullo et al. (2020a), Agullo et al. (2020b), Sreenath et al. (2019), Agullo et al. (2021a), Agullo et al. (2021b)]. In this paper, we use the procedure introduced in Ashtekar and Gupt (2017a), Ashtekar and Gupt (2017b). Strategy is to select these states by introducing some trial principles that relate properties of quantum geometry in the Planck regime with the late time geometry (which can be taken to be that given by general relativity to an excellent degree of approximation). One can then work out the observable consequences. If any prediction is ruled out by observations, one would return to the drawing board and seek alternate principles that would lead to viable states. If predictions are confirmed by observations, one would build confidence in the general direction and attempt to put the principles on a firmer and more satisfactory footing. The currently used principles are somewhat analogous to the Bohr model of the hydrogen atom in the early days of quantum mechanics. While in retrospect it is naive in some fundamental respects, nonetheless the Bohr model was useful because it captured some essential features of the final, correct description of the hydrogen model.

The first principle constrains $\Psi(a, \phi)$, determining the number of e-folds between the LQC bounce and the CMB surface (i.e., the surface of last scattering) (Ashtekar and Gupt, 2017b). One begins with the observation that the presence of a positive cosmological constant implies that there are cosmological horizons. As a result, given an instant of time there is a maximum value for the radius of a ball that any one observer can see, no matter how long she waits. In the standard $\Lambda$ CDM model, this radius is $\sim 17.29 \mathrm{Mpc}$ at the surface of last scattering. Thus, specification of initial data in this ball determines the entire future of the Universe that is accessible to an 'eternal' observer. As we go to the past, this ball shrinks and, interestingly, at the onset of inflation its physical radius is only $\sim 2.64 \times 10^{7} \ell_{\mathrm{Pl}}$ for both, the Starobinsky and quadratic potentials. This value is already smaller than the radius of a proton! At the bounce surface of LQC, its radius would be still smaller. From the LQG perspective, it is natural to require that the physical radius of the ball be $6 \Delta$, the minimum value allowed in the spatially flat FLRW quantum geometry. This requirement constrains $\Psi(a, \phi)$ such that the number $N_{\mathrm{B}-\mathrm{CMB}}$ of e-folds from the bounce to the surface of last scattering to be $\simeq 134$, or the number of e-folds from the bounce to today to be $\simeq 141$.

Finally, the principle that determines the quantum state $\psi(\mathcal{Q}, \phi)$ of scalar modes (Ashtekar and Gupt, 2017b) involves 
a quantum generalization of Penrose's Weyl curvature hypothesis (Penrose, 2004) in the Planck regime near the bounce, which physically corresponds to requiring that the state should be 'as isotropic and homogeneous in the Planck regime, as the Heisenberg uncertainty principle allows'. While the first principle sets the scale at which the LQC primordial spectrum ceases to be nearly scale invariant, and thus differs from that given by the $S A$, the second led to the conclusion that there is power suppression with respect to the $S A$ rather than power enhancement. Detailed calculations are needed to obtain the precise degree of suppression.

\section{RESULTS}

In this section we present the main results of this paper, obtained using the LQC summarized in Section 3. In terms of more commonly used wavenumbers, the new length scale $r_{\text {cuv }}^{\min } \sim 0.31 \ell_{\mathrm{Pl}}$ introduced by LQC provides a new physical scale $k_{\mathrm{LQC}} \simeq 3.21 \ell_{\mathrm{Pl}}^{-1}$, and the primordial spectrum differs from the $S A$ for $k_{\mathrm{phy}} \lesssim k_{\mathrm{LQC}}$ at the bounce. In Section 4.1 we first discuss these LQC corrections and then their effect on the observed $C_{\ell}^{X Y}$ correlations (where $X Y$ refers to TT, TE, EE and $\phi \phi)$. We also present predictions for the value of the optical depth $\tau$ and for the BB power spectrum that could potentially be tested using future observations. In Section 4.2, we show that the LQC predictions for the $C_{\ell}^{X Y}$ 's lead to resolution of power suppression and lensing amplitude anomalies. In Section 4.3, we will show that the interplay between LQC and observations is a 2-way bridge, in that the CMB observations can also be used to constrain the value of the area gap $\Delta$, the most important of fundamental microscopic parameters of LQG.

\subsection{Power Spectra}

As we saw at the end of Section 3, the physical principle used to select the background quantum geometry implies that the corresponding $\Lambda \mathrm{CDM}$ Universe has undergone approximately $141 \mathrm{e}$-folds of expansion since the quantum bounce until today (Ashtekar and Gupt, 2017b). Therefore, the characteristic physical scale $k_{\mathrm{LQC}}$ at the LQC bounce translates to the comoving wavenumber $k_{o} \simeq 3.6 \times 10^{-4} \mathrm{Mpc}^{-1}$ which sets the scale below which LQC corrections to the primordial scalar power spectrum become important. In particular, the calculations show that for scales $k \leqq 10 k_{o}$ the power is suppressed whereas for scales $k \gg k_{o}$ the power spectrum is essentially scale invariant as in the $S A .^{5}$ This behavior is captured in following modification to the $S A$ for the primordial power spectrum:

${ }^{4}$ This condition provides a small ball in the space of all quasi-free states and the desired state $\psi(\mathcal{Q}, \phi)$ is the one in this ball that is 'maximally classical' at the end of inflation in a specific, well-defined sense (Ashtekar and Gupt, 2017).

${ }^{5}$ The value of $k_{o}$ is linear in $k_{\mathrm{LQC}}$ and $10 k_{o} \simeq 3.6 \times 10^{-3} \mathrm{Mpc}^{-1}$ corresponds to $\ell \simeq 30$. Hence if one were to increase (or decrease) $k_{\mathrm{LQC}}$ by hand, the LQC effects would manifest themselves in observations at larger (respectively, smaller) values of $\ell$.

$$
\mathcal{P}_{\mathcal{R}}^{\mathrm{LQC}}(k)=f(k) A_{s}\left(\frac{k}{k_{\star}}\right)^{n_{s}-1}=f(k) \mathcal{P}_{\mathcal{R}}^{\mathrm{SA}}(k),
$$

where $f(k)$ is the correction factor (which is equivalent to the ratio of the power spectra in LQC to that in the $S A$ ).

The left panel of Figure 4 shows the correction factor $f(k)$ plotted with respect to the co-moving wavenumber. It is evident from the plot that for both the Starobinsky and quadratic potentials, relative to the prediction of the $S A$, there is power suppression for long wavelength modes corresponding to $k \leq 10 k_{o}$. The origin of this difference lies in the fact that, during the pre-inflationary era, the physical wavelength of these modes is sufficiently large to 'experience' the background curvature in the Planck regime near the bounce, leading to excitations over the standard Bunch-Davies (BD) state at the onset of inflation. On the other hand, the physical wavelengths of modes with $k \gg k_{o}$ are much smaller than the curvature radius throughout the pre-inflationary phase, including the deep Planck regime near the bounce. Therefore their state is practically the same as the standard BD state at the onset of inflation. Note that even for modes with $k \leq 10 k_{o}$, LQC corrections are significant only near the bounce. During this epoch the energy density of the scalar field is dominated by the kinetic term. Therefore one would expect the inflationary potentials to have negligible effect on the evolution of the background geometry and perturbations in the deep Planck regime, which is when the LQC corrections are imprinted on the modes of scalar perturbations. This expectation is explicitly borne out in the left panel in Figure 4: the primordial spectra for Starobinsky and quadratic potentials are essentially identical. Analytical considerations of (Bhardwaj et al., 2019) suggest that this feature will persist for a large class of inflationary potentials. Finally, note that the LQC primordial spectrum has a turnaround at very large wavelengths whose origin is related to the sudden spike in $r_{\text {curv }}$ near the bounce. Although these modes are not in the observable range for $\mathrm{CMB}$, it is of interest to better understand the origin of this growth in power for very small $k$ because, together with large and strongly scale dependent nonGaussianity (which is expected from the LQC bounce (Agullo et al., 2018; Sreenath et al., 2019) it could lead to a coupling between the long wavelength modes and the modes observable in $\mathrm{CMB}$, resulting in a modulation of the primordial power spectrum of the observable modes. Such a non-Gaussian modulation could explain the dipolar modulation of $\mathrm{CMB}$ and preference for odd parity that has been observed in the CMB (Agullo et al., 2021a; Agullo et al., 2021b).

Let us now turn to the LQC predictions for observable power spectra. For definiteness, in these plots we work with the Starobinsky potential for the $S A$ and LQC theoretical predictions. On the observational side, 'PLANCK 2018' refers to the $\mathrm{TT}+\mathrm{TE}+\mathrm{EE}+$ lowE + lensing 2018 data released by the PLANCK collaboration. In order to obtain constraints on the parameters in LQC and SA models, we used the publicly available software package COSMOMC (Lewis and Bridle, 2002) which supports the likelihood code used in the original 2018 PLANCK analyses. COSMOMC is based on the Markov-Chain-MonteCarlo (MCMC) procedure for estimation of parameters based on maximum likelihood analysis. For a given theoretical model with 

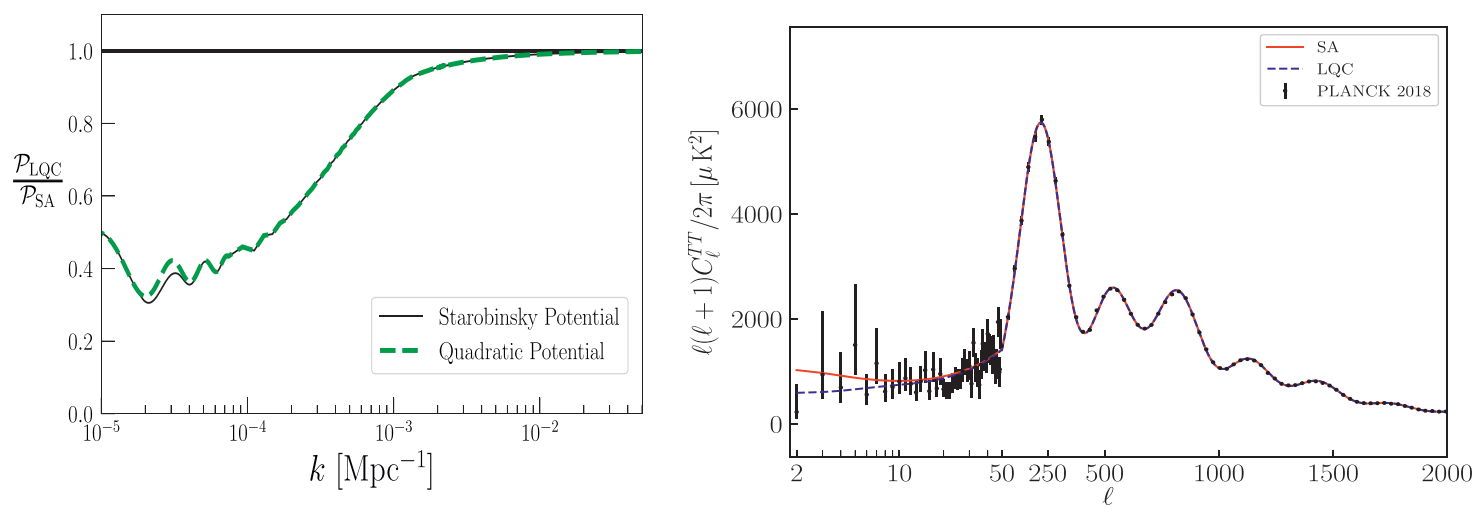

FIGURE 4 | Left Panel: The primordial power spectrum of LQC: The LQC suppression factor $f(k)$ is less than 1 for $k \leq 10 k_{\circ} \simeq 3.6 \times 10^{-3}$ Mpc ${ }^{-1}$ for both the Starobinsky and quadratic potentials. At short wavelengths (large $k$ ), or small angular scales, the LQC power spectrum is indistinguishable from the SA i.e. $f(k)=1$ for $k \gg k_{0}$. Right Panel: TT power spectra. The 2018 PLANCK spectrum (black dots with error bars), the LQC (dashed (blue) line) and the standard ansatz (SA) predictions (solid (red) line). As is usual, on the $x$-axis we have used a logarithmic scale for $\ell \leq 50$ and linear scale for $\ell>50$.
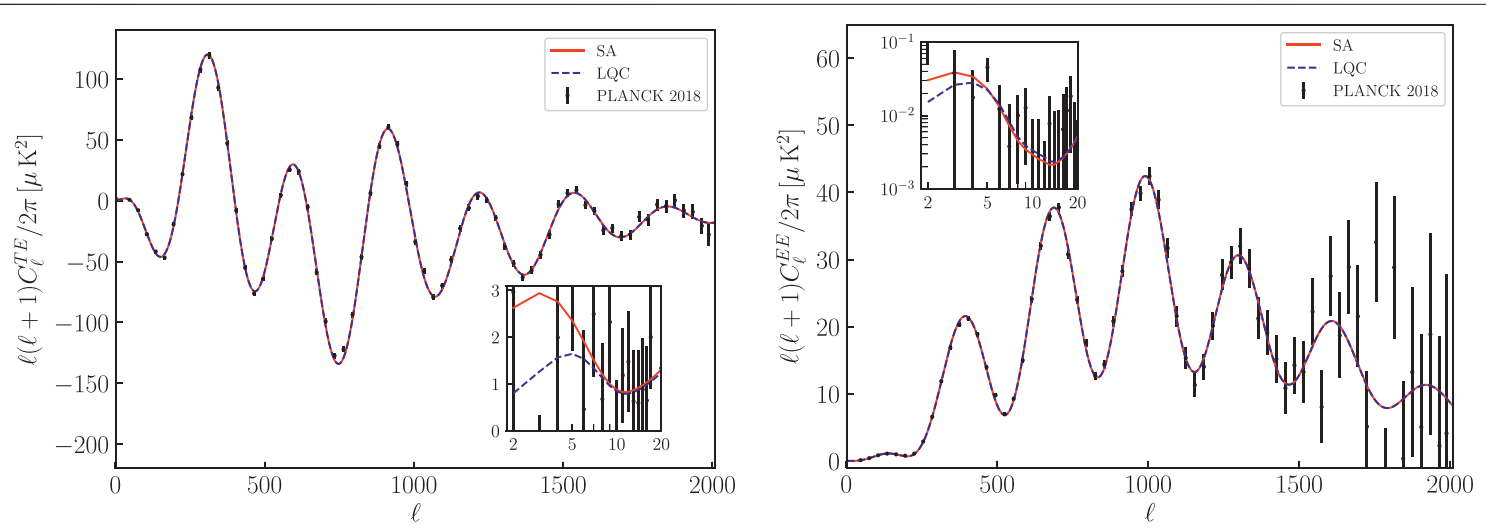

FIGURE 5 | TE and EE power spectra. The 2018 PLANCK spectrum (black dots with error bars), the LQC (dashed (blue) line) and the standard ansatz (SA) predictions (solid (red) line). Values of the $6 \Lambda$ CDM parameters are fixed to their best fit values shown in Table 1.

a number of parameters (here the $\Lambda \mathrm{CDM}$ model with 6 parameters), one builds a Markov chain from randomly sampled parameter values from a space predefined by priors. Each Markov chain begins with a random sample from the priorconstrained parameter space. Subsequent steps in the chain are then selected via Metropolis-Hasting algorithm: a Monte Carlo sample from the parameter space is proposed and then accepted or rejected based on the likelihood function which quantifies the degree of agreement between the theoretical prediction and the experimental data [in this paper we work with the same likelihood function as used in PLANCK 2018 papers (Aghanim et al., $2020 \mathrm{~b})]$. This procedure is repeated until a convergence criteria is satisfied. The chain of accepted values are further trimmed and thinned in order to remove dependence on the initial data point and correlation between subsequent steps. The converged Markov chain thus obtained approximates the posterior distribution of the parameters to be estimated. In order to obtain one-dimensional constraints on (or twodimensional correlation between) the individual parameters,
TABLE 1 | Comparison between the Standard Ansatz (SA) and LQC. The mean values of marginalized probability distributions for the six cosmological parameters, and values of $S_{1 / 2}$ calculated using $C_{\ell}^{\Pi}$.

\begin{tabular}{lcc}
\hline Parameter & SA & LQC \\
\hline$\Omega_{b} h^{2}$ & $0.02238 \pm 0.00014$ & $0.02239 \pm 0.00015$ \\
$\Omega_{C} h^{2}$ & $0.1200 \pm 0.0012$ & $0.1200 \pm 0.0012$ \\
$100 \theta_{M C}$ & $1.04091 \pm 0.00031$ & $1.04093 \pm 0.00031$ \\
$\tau$ & $0.0542 \pm 0.0074$ & $0.0595 \pm 0.0079$ \\
$\ln \left(10^{10} A_{S}\right)$ & $3.044 \pm 0.014$ & $3.054 \pm 0.015$ \\
$n_{S}$ & $0.9651 \pm 0.0041$ & $0.9643 \pm 0.0042$ \\
$S_{1 / 2}$ & $42,496.5$ & $14,308.05$ \\
$A_{L}$ & $1.072 \pm 0.041$ & $1.049 \pm 0.040$
\end{tabular}

marginalization procedure is used. For details of the MCMC techniques adapted to cosmological settings see (Lewis and Bridle, 2002).

The right panel of Figure 4 shows the theoretical predictions as well as the observed power spectrum, with its error bars. The 

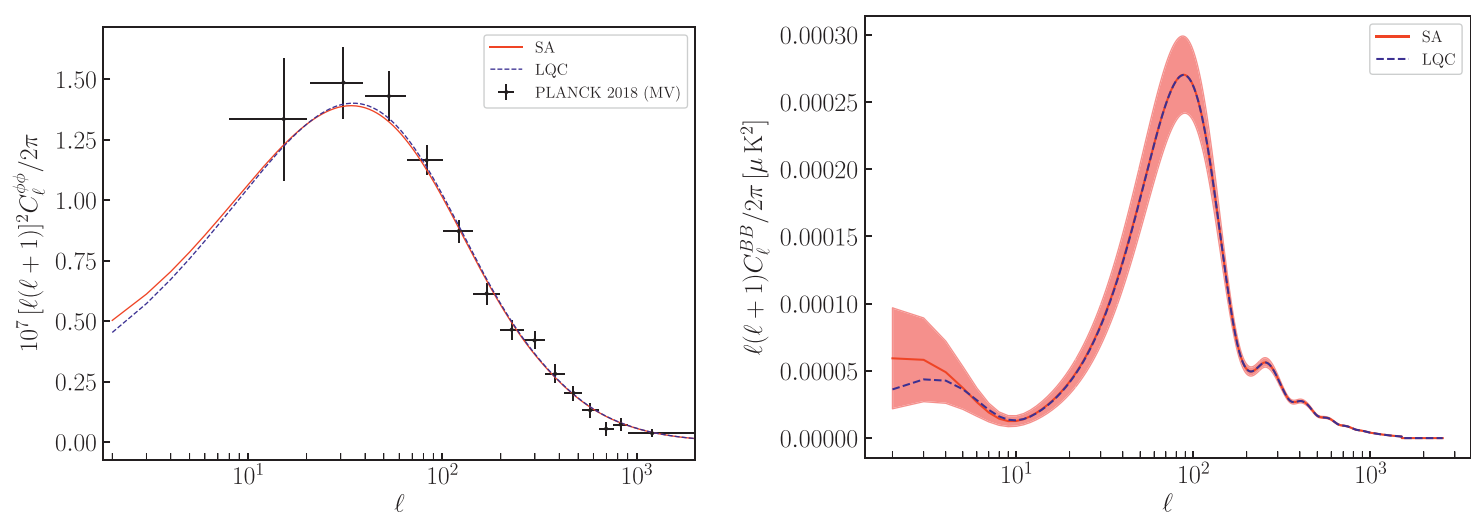

FIGURE 6 | LQC prediction (dashed (blue) line) and the standard ansatz (SA) prediction (solid (red) line). Left Panel: Lensing power spectra. The 2018 PLANCK spectrum (where the black crosses show binning and error bars). Right Panel: The theoretical predictions for the BB power spectra. There is power suppression in LQC for very low $\ell$. The shaded region shows the $68 \%$ confidence level region.

plots clearly show that the primordial power suppression of LQC for small $k$ leads to power suppression in the TT spectrum for multipoles $\ell<30$, relative to the predictions from the $S A$. The same primordial power spectrum can also be used to compute the predicted TE and EE correlation spectra. Figure 5 shows the TE (left panel) and EE (right panel) spectra as observed by Planck 2018, along with those obtained using the best fit $\Lambda$ CDMmodels with LQC and the $S A$. Note that in the right panel of Figure 4 and in Figure 5 the LQC and the SA plots are shown for their corresponding marginalized mean values of the 6 parameters shown in Table 1. By contrast, in (Ashtekar and Gupt, 2017b) both the LQC and the $S A$ curves were plotted for the same values of 6 parameters of the $\Lambda \mathrm{CDM}$ model, coming from the $S A$. As Table 1 shows, the mean value of optical depth $\tau$ is $>9 \%$ higher in LQC than with the $S A$ and electric polarization is quite sensitive to $\tau$. Interestingly, this change in the best fit $\Lambda$ CDM Universe has the effect of slightly reducing suppression in the passage from the primordial power spectrum to the observed one. As a result, the LQC power suppression in the TT, TE and EE spectra we now find is somewhat less pronounced than it was in Ashtekar and Gupt (2017b).

Finally, the left panel of Figure 6 shows the lensing correlation spectrum $C_{\ell}^{\phi \phi}$ reported by the PLANCK collaboration with their 2018 data, along with the LQC and the $S A$ predictions. For this figure we have again used the mean marginalized values of the 6 parameters shown in Table 1, and the lensing amplitude is fixed to $A_{L}=1$, in accordance with the base $\Lambda \mathrm{CDM}$ model. As with the TT, TE and EE spectra, the lensing spectrum also shows suppression at large angular scales corresponding to $\ell<30$. However, observations are quite sparse for low $\ell$. Interestingly, for $30<\ell<100$, the LQC prediction for $C_{\ell}^{\phi \phi}$ is slightly larger than that for SA (This is in fact inevitable; see Section 5). This behavior leads the LQC spectrum to fit slightly better with the observed data in the range $30<\ell<100$ and hints toward resolving the lensing amplitude anomaly in LQC without having to introduce additional modifications to the standard $\Lambda \mathrm{CDM}$ model. In the next subsection we will see that this possibility is in fact realized.
We will conclude this discussion of observable implications of LQC with predictions for future missions. First, as we noted in Section 2.2, currently the optical depth $\tau$ is the least accurately measured of the $6 \Lambda \mathrm{CDM}$ parameters, with a relative error of $\sim 13 \%$. The LQC value is some $9.8 \%$ higher than that in the Universe according to PLANCK'. This prediction will be tested by the future observation of global $21 \mathrm{~cm}$ evolution at high redshifts that is estimated to reach a percent level accuracy in the measurement of $\tau$ (Fialkov and Loeb, 2016). As for the observable power spectra, to date the PLANCK satellite has provided the best full sky measurement of the CMB anisotropies. However, there is still scope for improvement in the measurement of electric polarization, and the odd-parity magnetic polarization is yet to be detected. Therefore, several space-based mission have been planned to further improve the polarization measurements. In addition to the predictions for the TE and EE power spectra discussed above, our LQC model also makes predictions for the $\mathrm{BB}$ power spectrum. The right panel of Figure 6 provides the prediction for the unlensed BB power spectrum both from the $S A$ and in LQC. Recall that the tensor-toscalar ratio $r$ depends on the potential of the inflationary model. But, being a ratio, it is the same in LQC as from SA (Agullo et al., 2013b). We have set $r=0.0041$, its value for the Starobinsky potential. As in the case of other four spectra, we observe a relative suppression of power at low multipoles. However, this is also where the reionization bump occurs. Since LQC predicts a larger value of optical depth, the B-B power suppression is lower than what one might have expected from the primordial power suppression (and using the same value of $\tau$ for both SA and LQC). Nonetheless, it may be possible to test this prediction against the data from the future B-mode missions such as LiteBIRD (Matsumura and et al., 2014), Cosmic Origins Explorer (Delabrouille and et al., 2018), $\mathrm{ECHO}^{6}$ or Probe Inflation and Cosmic Origins (PICO) (Hanany et al., 2019) (which should observe the $\mathrm{BB}$ spectrum if $r \gtrsim 0.001$ ).

${ }^{6} \mathrm{http}: / / \mathrm{cmb}$-bharat.in/ 


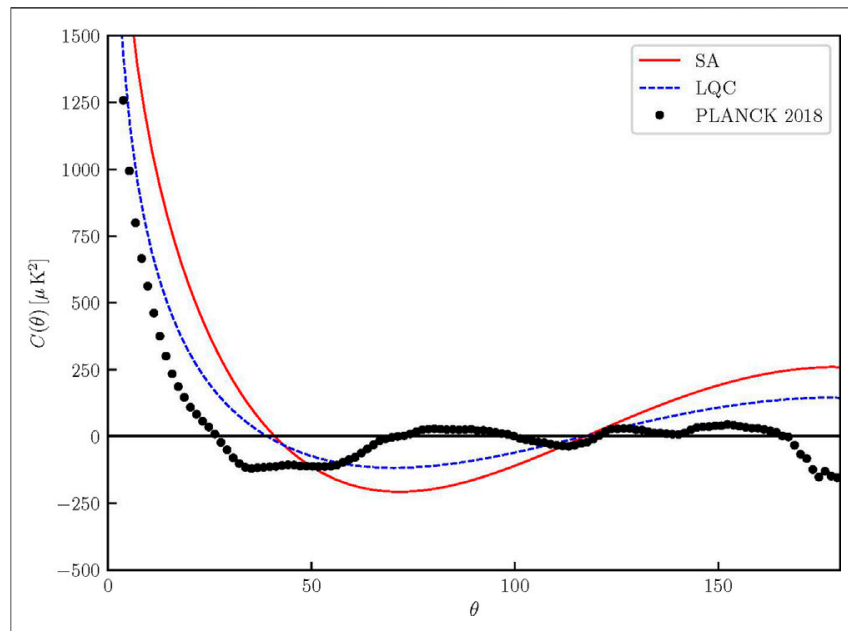

FIGURE 7 | The temperature-temperature angular power spectrum $C(\theta)$. The 2018 PLANCK spectrum (thick black dots), the LQC [dashed (blue) line], and the standard ansatz [solid (red) line] predictions. The LQC predictions are closer to the observed Planck 2018 data points.

\subsection{Anomalies Alleviated}

As discussed in Section 2.2, in our approach the pre-inflationary phase of LQC dynamics enables one to address two CMB anomalies. The first, shown in the left panel of Figure 2, is that the observed angular correlation function $C^{\mathrm{TT}}(\theta)$ remains close to zero for angles $\theta>60^{\circ}$ in contrast to the predictions of the standard $\Lambda C D M$ model based on the $S A$. Let us now examine the prediction of $C^{\mathrm{TT}}(\theta)$ from LQC. This prediction is plotted as the dashed (blue) curve in Figure 7, along with the prediction from the $S A$ shown as the (red) dashed curve and the $C^{\mathrm{TT}}(\theta)$ observed by the 2018 PLANCK mission shown by (black) dots. One sees by inspection that the LQC predictions are closer to the observed values than the $S A$ predictions. This behavior can be further quantified by comparing the value of $S_{1 / 2}:=\int_{-1}^{1 / 2}[C(\theta)]^{2} d(\cos \theta)$ which, as we saw in Section 2.2, represents a cumulative total power at large angular scales $\left(\theta>60^{\circ}\right)$ :

$$
S_{1 / 2}^{\text {Planck }}=1209.18 ; \quad S_{1 / 2}^{\mathrm{SA}}=42496.5 ; \quad S_{1 / 2}^{\mathrm{LQC}}=14308.05 .
$$

The tension between observations and the theoretical prediction from the $S A$ is encapsulated by the fact that $S_{1 / 2}^{S A}$ is almost 35 times larger than $S_{1 / 2}^{\text {Planck }}$. This is the power suppression anomaly. This discrepancy is appreciably reduced in LQC since $S_{1 / 2}^{\mathrm{LQC}}$ is $\sim 1 / 3$ of $S_{1 / 2}^{\mathrm{SA}}$. As indicted in Section 2.2, because $C(\theta)$ for different values of $\theta$ are correlated, and because one has to use a masking procedure in the data analysis near $\theta=180^{\circ}$, the task of providing the $1 \sigma$ and $2 \sigma$ contours around the LQC plots is challenging, requiring manipulations of a large covariance matrix in the data analysis, as well as a detailed understanding of aspects of the instrument. It would be of considerable interest if the $\mathrm{CMB}$ experts could provide these plots starting with the LQC TT-power spectrum. Note also that because power is suppressed for $\ell \leqq 30$, if LQC results were used in the PLANCK analysis, error bars for low $\ell$ would also

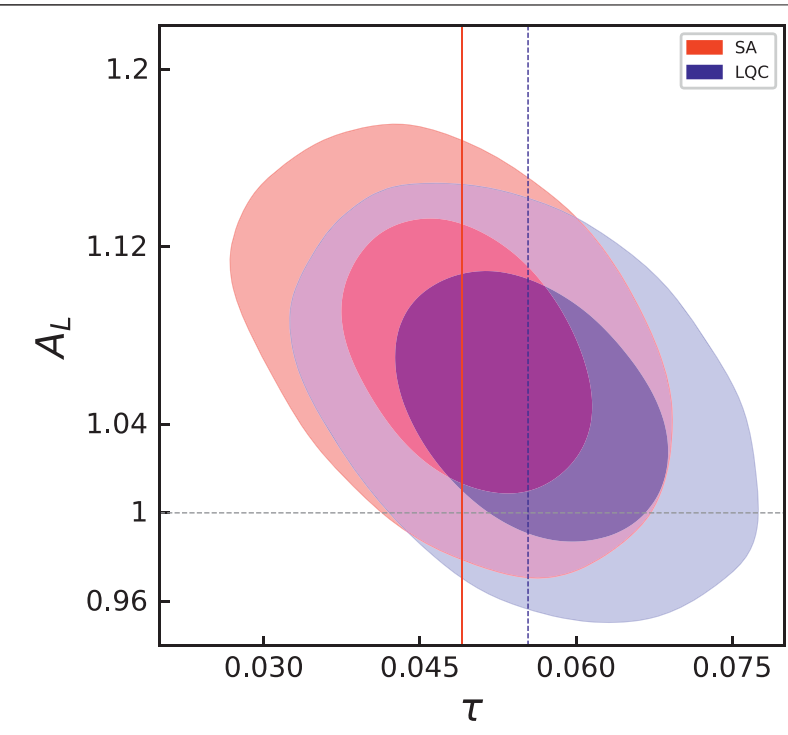

FIGURE 8 | $1 \sigma$ and $2 \sigma$ probability distributions in the $A_{L}-\tau$ plane. Predictions of the $S A$ (in red) and LQC (in blue). Vertical lines denote the mean values of $\tau$ for the $S A$ and LQC. It is evident that $A_{L}=1$ is outside the $1 \sigma$ contour for the $S A$ while is restored within $1 \sigma$ for the LQC model.

be reduced and we would have a sharp measure of the LQC alleviation of this anomaly.

The second anomaly, shown in the right panel of Figure 2, is the lensing amplitude anomaly. This anomaly is not directly observed in the $C_{\ell}^{\mathrm{XX}}$ plots or in $C(\theta)$ plots, but arises when one performs a consistency check of the $\Lambda$ CDM model. Instead of fixing the lensing amplitude to $A_{L}=$ 1 (as is assumed in standard $\Lambda$ CDM model) one allows it to vary along with the standard 6 parameters, i.e., one now analyzes a 7 parameter model. The anomaly lies in the finding that $A_{L}=1$ is beyond $1 \sigma$ error bar as shown in the right panel of Figure 2. This led the authors of Di Valentino et al. (2019) to conclude that there is a possible "crisis in cosmology" because, to alleviate this problem, one would need to introduce spatial curvature which creates significant departures from the observed power spectra as small angular scales. As shown in Figure 8, however, repeating the analysis with the LQC power spectrum restores $A_{L}=1$ within $1 \sigma$ contour thereby resolving the lensing amplitude anomaly and avoiding the hint of a potential "crisis".

To summarize, the observed CMB power spectrum has many non-trivial and interesting features at small angular scales and observational error bars are small in this regime. It is remarkable that these features are correctly predicted by the $\Lambda$ CDM model based on the SA. The LQC corrections could well have led to discrepancies with these successful predictions of the $S A$. That does not happen. Rather, there are departures only at large angular scales leading to a suppression of the primordial power for small $k$. This difference from the nearly scale invariant standard ansatz (2.1) leads to the alleviation of two anomalies in the CMB. 


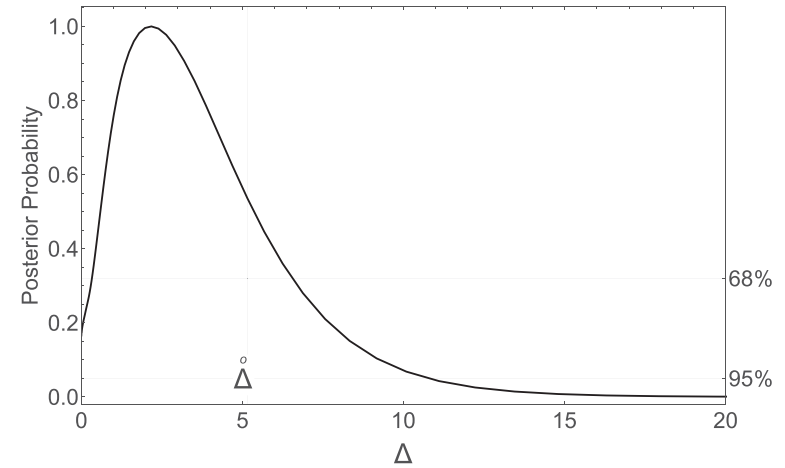

FIGURE 9 | Marginalized posterior probability distribution for $\Delta$. The value $\tilde{\Delta} \simeq 5.17 \ell_{\mathrm{P} \mid}^{2}$ selected by the black hole entropy considerations is denoted by the vertical line. It lies within $1 \sigma$ of the marginalized mean value $3.86 \ell_{\mathrm{PI}}^{2}$ that lies to the right of the peak. Thus, there is an unforeseen internal consistency.

\subsection{From Observations to Fundamental Theory}

As we saw in Section 3, the area gap $\Delta$ is the key microscopic parameter that determines values of important new, macroscopic observables such as the matter density and the curvature at the bounce. Its specific value, $\tilde{\Delta}=5.17 \ell_{\mathrm{Pl}}^{2}$, is determined by the statistical mechanical calculation of the black hole entropy in loop quantum gravity [see, e.g. Ashtekar et al. (1998), Ashtekar et al. (2000), Barbero and Perez (2017), Perez (2017)]. Results reported in the last two subsections are based on this value. However, with the CMB observations at hand, we can now, so to say, turn the tables and regard $\Delta$ as an additional parameter and use the $C M B$ observations to constrain its value.

Thus, let us consider $\Delta$ to be a free parameter and obtain a posterior probability distribution for its value by letting it vary along with the 6 parameters of the $\Lambda \mathrm{CDM}$ model. Note that this procedure is similar to the self-consistency check that is performed for the lensing amplitude $A_{L}$. The $\Lambda \mathrm{CDM}$ Universe determined by the $S A$ does not pass that test because, when it is allowed to vary, $A_{L}$ prefers a value that is greater than 1 and the discrepancy is significant in that the value 1 lies outside the $1 \sigma$ contour of the best fit value (Adam et al., 2020). Is there perhaps a similar tension here? More precisely, does the value $\tilde{\Delta} \simeq 5.17 \ell_{\mathrm{Pl}}^{2}$ obtained from black hole entropy calculations lie within the $68 \%$ confidence contour of the value preferred by the $\mathrm{CMB}$ observations? If it does not, LQC would fail the self-consistency test at the $1 \sigma$ level. If it does, there would be an unforeseen coherence between detailed conclusions drawn from very different considerations: the LQG analysis of black hole entropy and the LQC investigation of the very early Universe!

Figure 9 shows the one-dimensional posterior distribution of $\Delta_{\mathrm{B}}$. The best-fit value-the peak in the distribution-is at $\Delta=$ $2.18 \ell_{\mathrm{Pl}}^{2}$ while the marginalized mean value is $3.86 \ell_{\mathrm{Pl}}^{2}$.with the following constraint:

$$
1.26 \ell_{\mathrm{Pl}}^{2}<\Delta<6.47 \ell_{\mathrm{Pl}}^{2} \quad(\text { at } 68 \% \text { confidence level })
$$

Clearly, the value $\tilde{\Delta} \simeq 5.17 \ell_{\mathrm{Pl}}^{2}$ chosen in Section 3.3 and used in this paper, is within $68 \%(1 \sigma)$ confidence level of the constraint obtained from Planck 2018. This not only indicates a synergy between the fundamental theoretical considerations and observational data, but also provides internal consistency of the LQC model.

\section{DISCUSSION}

To determine the six parameter $\Lambda \mathrm{CDM}$ Universe we live in, the PLANCK team began with the standard ansatz (2.1) for the primordial spectrum and used known astrophysics to determine the observable power spectra for various values of the six cosmological parameters, $A_{s}, n_{s}, \Omega_{b} h^{2}, \Omega_{c} h^{2}, 100 \theta_{M C}$. By comparing these theoretical predictions with observations, they determined the marginalized mean values of the six parameters (together with the error bars corresponding to 68\% confidence level). This is the $\Lambda$ CDM Universe according to PLANCK. This procedure has had tremendous success, especially with the finer features of the power spectra at small angular scales. However, there are also some anomalies. Since they are only at $\sim 2-3 \sigma$ level, the statistical significance of any one anomaly is low. However, taken together, two or more anomalies imply that we live in an exceptional realization of the six posterior distributions provided by this procedure.

One can view these anomalies as potential gates to new physics. Indeed, the PLANCK collaboration has emphasized this possibility in its 2015 (Ade et al., 2016) as well as 2018 data releases (Aghanim et al., 2020a). As the second of these papers points out, “. . if any anomalies have primordial origin, then their large scale nature would suggest an explanation rooted in fundamental physics. Thus it is worth exploring any models that might explain an anomaly (even better, multiple anomalies) naturally, or with very few parameters." LQC researchers have followed up on this suggestion. In this paper we presented a concrete realization of this idea. Specifically, one begins with the observation that, in the standard procedure summarized above, the theoretical input, beyond known astrophysics, is the $S A$, motivated by the inflationary scenario. It assumes that the primordial spectrum is nearly scale invariant and can be characterized just by two numbers $A_{s}$ and $n_{s}$ across all wavenumbers $k$. However, in LQC the resolution of the big bang singularity introduces a new scale $k_{\mathrm{LQC}}$ and quantum gravity corrections in the pre-inflationary phase of dynamics appear for $k \leq k_{\mathrm{LQC}}$. For these small wavenumber modes, the LQC primordial spectrum is no longer nearly scale invariant, whence there is departure from the predictions drawn from the $S A$.

Several closely related approaches have been used in LQC to probe the effects of this pre-inflationary dynamics [see, e.g. Agullo et al. (2012), Agullo et al. (2013a), Agullo et al. (2013b), Fernandez-Mendez et al. (2012), Barrau et al. (2014), Linsefors et al. (2013), Ashtekar and Barrau (2015), Agullo and Morris (2015), Agullo (2015), Ashtekar and Gupt (2017a), Ashtekar and Gupt (2017b), Gomar et al. (2017), Agullo et al. (2018), Barrau et al. (2018), Sreenath et al. (2019), Agullo et al. (2021b)]. Our approach has two main ingredients: 1) the use of sharply peaked quantum states $\Psi(a, \phi)$ for the background quantum FLRW geometry, that then enable one 
to well-approximate the dynamics of cosmological perturbations on the quantum geometry $\Psi(a, \phi)$ by that on a quantum corrected 'dressed metric' $\tilde{g}_{a b}$ (Ashtekar et al., 2000; Agullo et al., 2013b); and, 2) the use of certain principles to select $\Psi(a, \phi)$ and the quantum state $\psi(\mathcal{Q}, \phi)$ of the scalar mode of cosmological perturbations for any given inflationary potential (Ashtekar and Gupt, 2017a; Ashtekar and Gupt, $2017 b)$. For any given inflationary potential, the principle used to select $\Psi(a, \phi)$ limits the number of e-folds during pre-inflationary dynamics, thereby implying that the modes that receive significant LQC corrections in the primordial spectrum correspond to the large angular scales $\ell \leqq 30$. The principle used to select $\psi(\mathcal{Q}, \phi)$ implies that in the primordial spectrum there is power reduction (rather than enhancement) in these modes. This then translates to a power suppression for $\ell \leqq 30$ in the observed power spectra. For modes with $\ell \gg 30$, the LQC power spectra are indistinguishable from those obtained using the $S A$. Thus, LQC predictions leave the highly successful predictions of standard inflation at small angular scales unaffected, but modify the predictions at large angular scales.

Details of the LQC pre-inflationary dynamics reveal some interesting facts. First, the quantum geometry effects on the background FLRW geometry are dominant only in a short interval around the bounce. Second, it is the modes whose physical wavelength $\lambda_{\text {phy }}$ is longer than the curvature radius $r_{\text {curv }}$ during the pre-inflationary evolution that fail to be in the $B D$ vacuum at onset of inflation. In the observable band, only the longest wavelength modes are thus affected and they satisfy $\lambda_{\text {phy }} \gtrsim r_{\text {curv }}$ only for $2-3$ e-folds after the bounce. Thus, the background quantum geometry as well as the quantum perturbations Planck receive non-negligible LQC corrections during a very short duration. Yet these corrections lead to observable effects in that they alleviate some anomalies. Third, while we did not discuss tensor modes in this paper, their power spectra have the same behavior as that of scalar modes and, given an inflationary potential, the tensor to scalar ratio $r$ does not receive LQC corrections (within accuracies reported here). Finally, there is an unforeseen interplay between the UV and the IR: While it is the $U V$ modifications of GR that lead to the singularity resolution and create the new LQC scale $k_{\mathrm{LQC}}$, the structure of the evolution equations satisfied by cosmological perturbations is such that it is the $I R$ modes with $k \leqq k_{\mathrm{LQC}}$ that are affected during their pre-inflationary evolution. It is this unforeseen cosmic tango between the very small and the very large that is responsible for the alleviation of the two anomalies discussed in this paper.

Given that LQC simultaneously alleviates the power suppression and the lensing amplitude anomalies, it is worth investigating a more general question: Are the two conceptually related? As reported in Ashtekar et al. (2020), the answer is in the affirmative. Since this relation seems not to have been noticed before, we will make a small detour to explain it. Let us begin by assuming that there is some mechanism-not necessarily originating in LQC-that provides a primordial power spectrum of the form

$$
\mathcal{P}_{\mathcal{R}}^{\text {new }}(k)=f(k) A_{s}\left(\frac{k}{k_{\star}}\right)^{n_{s}-1}
$$

with $f(k)<1$ for $k<k_{\circ}$ and $f(k)=1$ for $k>k_{\circ}$ for some $k_{\circ}$, and compare and contrast the new $\Lambda$ CDM Universe obtained from this modified ansatz with that given by the SA of Eq. 2.1. In the first step, we can restrict our analysis only to smaller angular scales $\left(k \gg k_{\circ}\right)$. Then, the primordial spectrum in both schemes would be the same, whence we would obtain the same marginalized mean values of the six cosmological parameters. Denote by $\AA_{s}$ the marginalized mean value of the scalar amplitude $A_{s}$ thus obtained. In the second step, let us consider the full range of observable modes, including $k \leq k_{\circ}$. Now, given that the observations show that the TT power is suppressed at largescales, i.e., for $k \lesssim k_{\circ}$, if one uses the $\mathrm{SA}+\Lambda \mathrm{CDM}$ model the marginalized mean value $A_{s}^{\mathrm{SA}}$ using the entire $k$ range will be lower than $\AA_{s}$. By contrast if the primordial power spectrum is of the form of Eq. 5.1, the initial power is already suppressed by $f(k)$. Therefore, $\AA_{s}$ will not have to be lowered as much to obtain the marginalized mean value $A_{s}^{\text {new }}$. Thus, we have

$$
\stackrel{\circ}{A}_{s}>A_{s}^{\text {new }}>A_{s}^{\mathrm{SA}}
$$

The key point is the last inequality: $A_{s}^{\text {new }}>A_{s}^{\mathrm{SA}}$ (We spelled out the argument because at first it seems counter-intuitive that power suppression leads to a larger $A_{s}^{\text {new }}$. But note that power is suppressed only for low $k$.). Next, we know that for large $k$, the product $A_{s} e^{-2 \tau}$ is fixed by observations. Hence, it follows that the best fit values of the optical depth in the two scheme must satisfy $\tau^{\text {new }}>\tau^{\mathrm{SA}}$. Finally, from the very definition of lensing amplitude, the value of $A_{L}$ is anti-correlated to the value of $A_{s}$. Therefore, it follows that we have the inequality $A_{L}^{\text {new }}<A_{L}^{\text {SA }}$. Thus in any theory that has primordial spectrum of the form (5.1), $A_{s}, \tau$ and $A_{L}$ will have the same qualitative behavior as in LQC, and hence the tension with observations would be reduced. What LQC provides is a precise form of the suppression factor $f(k)$ from 'first principles', and hence specific quantitative predictions. Also, recall from Section 4.1 that in our analysis the LQC $f(k)$ also came with a specific value $k_{o} \simeq 3.6 \times 10^{-4} \mathrm{Mpc}^{-1}$ for $k_{\text {。 }}$. Other mechanisms could well lead to a very different value. If so, in the observed power spectra suppression would arise at a very different value of $\ell$. Finally, the specific $f(k)$ computed from LQC also leads to other predictions-e.g., for the BB power spectrum discussed in Section 4.1-that need not be shared by other mechanisms.

In this respect, it would be of interest to compare the LQC predictions with those that result from effective field theories à la Ginsburg and Landau, where slow roll inflation is generically preceded by a fast roll phase that leads to a suppression of CMB quadrupole (Boyanovsky et al., 2009). This will require a calculation of angular power spectrum $C(\theta)$, and of the measure $S_{1 / 2}$ of power suppression, using the marginalized posterior probabilities of the 6 cosmological parameters in this effective field theory approach, and a reanalysis of the lensing amplitude along the lines of Section 4.2. Yet other mechanisms have been proposed to account for power suppression at large angular scales in the context of GR [see, e.g. Kofman and 
Starobinsky (1985), Das and Souradeep (2014), Contaldi et al. (2003), Cline et al. (2003), Jain et al. (2009), Pedro and Westphal (2014), Lello et al. (2014), Cai et al. (2016)]. These are compared and contrasted with LQC in Section 5 of Ashtekar and Gupt (2017b). Finally power suppression at large angular scales has been studied in the context of other bouncing models. In those discussions the bounce is often just assumed [as in Cai et al. (2009)], or obtained by adding a scalar field with a negative kinetic term which violates standard energy conditions [as in Liu et al. (2012)]. Our approach is different in that: 1) the bounce is a prediction of LQC; 2) since the mechanism has its roots in quantum geometry effects underlying LQG, additional scalar fields or violations of energy conditions are not involved; 3) the standard inflationary potentials are used without adjustments to provide a fast roll phase; and, most importantly, 4) our goal is to investigate whether the $\mathrm{CMB}$ observations can inform quantum gravity and vice versa.

In our view, the big bounce and the pre-inflationary dynamics of cosmological perturbations are on a robust footing although the discussion would benefit from a further sharpening of the detailed arguments that led us to the dressed metric $\tilde{g}_{a b}$. The part of the analysis that is on a less solid footing concerns the specific principles (Ashtekar and Gupt, 2017a; Ashtekar and Gupt, $2017 b)$ that were used to select the wave function $\Psi_{o}(a, \phi)$ of the background quantum geometry and the state $\psi(a, \phi)$ of perturbations. Note that these choices are necessary to make predictions in any approach that starts in the Planck regime. Indeed, even in standard inflation one has to assume that the perturbations are in the $\mathrm{BD}$ vacuum at the start of slow roll, and as discussed in Section 3.3, it is difficult to justify this assumption from first principles. The fact that the principles led us to predictions that not only reproduce the successes of standard inflation, but also alleviate the tension associated with two anomalies, is an indication that they set us on the right track. However, they should be regarded as tentative first steps, to be improved upon and sharpened in future. In particular, the effect of the duration of the reheating epoch is yet to be investigated in detail. Another direction for future work is suggested by a second approach that has been used to alleviate $\mathrm{CMB}$ anomalies using pre-inflationary dynamics (Sreenath et al., 2019; Agullo et al., 2021b). The point of departure is the same as in our approach: the bounce sets a new scale and modes with the longer wavelength at the bounce get excited during the pre-inflationary evolution and are no longer in the $\mathrm{BD}$ vacuum at the onset of inflation. However, the primordial spectrum does not exhibit power suppression (4.1) as in our case. Instead, a key role is played by the superhorizon modes and their non-Gaussian correlations with the longest wavelength modes with those that are observable in the CMB. These correlations enhance the probability of finding certain features in the individual realizations of the primordial probability distribution, thereby alleviating anomalies associated with dipolar asymmetry and power suppression anomalies. It would be of considerable interest to re-examine all $\mathrm{CMB}$ anomalies from a perspective that combines strengths of the two approaches. Finally, all approaches to finding observable consequences of LQC in $\mathrm{CMB}$, that we are aware of, assume an inflationary potential. At a certain level of discussion this is justified since a detailed analysis of the criticisms of the inflationary paradigm has concluded that, although important questions remain, the case for inflation has been "strengthened by the PLANCK data" (Chowdhury et al., 2019). However, from a fundamental perspective, introduction of an inflaton and a specific potential is ad-hoc. Now, in full LQG, the EinsteinHilbert Lagrangian receives higher order (in particular $R^{2}$ ) corrections because the curvature operator is constructed from Planck scale Wilson loops and hence non-local at the fundamental microscopic scale. Therefore it is important to analyze if these corrections would provide a natural basis for inflation purely from gravitational considerations, as in Starobinsky inflation.

\section{DATA AVAILABILITY STATEMENT}

Publicly available datasets were analyzed in this study. This data can be found here: Planck Satellite collaboration 2018 Data release.

\section{AUTHOR CONTRIBUTIONS}

AA Primary Contribution: Conceptual and Mathematical Framework (Sections 1, 2, 3, 5), BG and VS Primary Contribution: High performance computing to extract observable predictions from LQC (Section 4, and all the plots in the paper).

\section{FUNDING}

NSF grants PHY-1806356 and PHY-1505411; Penn State endowment funds associated with the Eberly Chair.

\section{ACKNOWLEDGMENTS}

We are grateful to Donghui Jeong for numerous discussions on observational aspects discussed in this paper and for collaboration in Ashtekar et al. (2020). We would like to thank Ivan Agullo, Francois Bouchet, Woiciech Kaminski, Jerzy Lewandowski, Charles Lawrence, Jerome Martin and Patrick Peter for valuable discussions and Pawel Bielewicz for help with Figure 7. One of the referee reports led to a clarifying remark at the end of Section 3, and another led to corrections of three typos that could have unnecessarily confused non-experts. This work was supported in part by the NSF grants PHY-1505411 and PHY-1806356, and the Eberly research funds of Penn State. Portions of this research were conducted with high performance computing resources provided by Louisiana State University (http://www.hpc.lsu.edu). 


\section{REFERENCES}

Adam, R., Aghanim, N., Akrami, Y., Ashdown, M., Aumont, J., Baccigalupi, M., et al. Planck Collaboration (2020). Planck 2018 Results VI. Cosmological Parameters. Astron. Astrophys. 641, A6.

Ade, P. A. R., Aghanim, N., Akrami, Y., Aluri, P. K., Arnaud, M., Ashdown, J., et al. Planck Collaboration (2016). Planck 2015 Results. XVI. Isotropy and the Statistics of the CMB. A\&A 594, A16. doi:10.1051/0004-6361/ 201526681

Aghanim, N., Akrami, Y., Ashdown, M., Aumont, J., Baccigalupi, M., Ballardini, M., et al. Planck Collaboration (2020a). Planck 2018 Results. I. Overview and the Cosmological Legacy of Planck". Astron. Astrophys. 641, A1. doi:10.1051/ 0004-6361/201833880

Aghanim, N., Akrami, Y., Arroja, F., Ashdown, M., Aumont, J., Baccigalupi, M., et al. Planck Collaboration (2020b). Planck 2018 Results. V. CMB Power Spectra and Likelihoods". Astron. Astrophys. 641, A5. doi:10.1051/0004$6361 / 201936386$

Agullo, I., Ashtekar, A., and Nelson, W. (2012). A Quantum Gravity Extension of the Inflationary Scenario. Phys. Rev. Lett. 109, 251301. doi:10.1103/physrevlett. 109.251301

Agullo, I., Ashtekar, A., and Nelson, W. (2013a). Extension of the Quantum Theory of Cosmological Perturbations to the Planck Era. Phys. Rev. D87, 043507. doi:10.1103/PhysRevD.87.043507

Agullo, I., Ashtekar, A., and Nelson, W. (2013b). The Pre-inflationary Dynamics of Loop Quantum Cosmology: Confronting Quantum Gravity with Observations. Class. Quan. Grav. 30, 085014. doi:10.1088/0264-9381/30/8/085014

Agullo, I., Bolliet, B., and Sreenath, V. (2018). Non-Gaussianity in Loop Quantum Cosmology D97, 066021. doi:10.1103/PhysRevD.97.066021

Agullo, I., Kranas, D., and Sreenath, V. (2021a). Anomalies in the CMB from a Cosmic Bounce. Gen. Rel. Grav. 53 (no.2), 17. doi:10.1007/s10714-020-02778-9

Agullo, I., Kranas, D., and Sreenath, V. (2021b). Large Scale Anomalies in the CMB and Non-Gaussianity in Bouncing Cosmologies. Class. Quant. Grav. 38 (no.6), 065010. doi:10.1088/1361-6382/abc521

Agullo, I. (2015). Loop Quantum Cosmology, Non-Gaussianity, and CMB Power Asymmetry. Phys. Rev. D 92, 064038. doi:10.1103/physrevd.92.064038

Agullo, I., and Morris, N. A. (2015). Detailed Analysis of the Predictions of Loop Quantum Cosmology for the Primordial Power Spectra. Phys. Rev. D 92, 124040. doi:10.1103/physrevd.92.124040

Agullo, I., Olmedo, J., and Sreenath, V. (2020a). Observational Consequences of Bianchi I Spacetimes in Loop Quantum Cosmology. Phys. Rev. D 102 (no.4), 043523. doi:10.1103/physrevd.102.043523

Agullo, I., Olmedo, J., and Sreenath, V. (2020b). Predictions for the Cosmic Microwave Background from an Anisotropic Quantum Bounce. Phys. Rev. Lett. 124 (no.25), 251301. doi:10.1103/physrevlett.124.251301

Agullo, I., and Parker, L. (2011a). Stimulated Creation of Quanta during Inflation and the Observable Universe. Gen. Rel. Grav. 43, 2541. doi:10.1007/s10714-0111220-8

Agullo, I., and Parker, L. (2011b). Non-gaussianities and the Stimulated Creation of Quanta in the Inflationary Universe. Phys. Rev. D 83, 063526. doi:10.1103/ physrevd.83.063526

Akrami, Y., Ashdown, M., Aumont, J., Baccigalupi, M., Ballardini, M., Banday, A. J., et al. Planck Collaboration (2019). Planck 2018 Results. VII. Isotropy and Statistics of the CMB. arXiv:1906.02552[astro-ph.CO].

Agullo, I., and Singh, P. (2017). "Loop Quantum Cosmology", in Loop Quantum Gravity: The First 30 Years, ed. A. Ashtekar and J. Pullin (Singapore: World Scientific).

Ashtekar, A., Baez, J., Corichi, A., and Krasnov, K. (1998). Quantum Geometry and Black Hole Entropy. Phys. Rev. Lett. 80, 904-907. doi:10.1103/physrevlett. 80.904

Ashtekar, A., Baez, J. C., and Krasnov, K. (2000). Quantum Geometry of Isolated Horizons and Black Hole Entropy. Adv. Theor. Math. Phys. 4, 1-94. doi:10. 4310/atmp.2000.v4.n1.a1

Ashtekar, A., and Barrau, A. (2015). Loop Quantum Cosmology: From Preinflationary Dynamics to Observations. Class. Quan. Grav. 32, 234001. doi:10.1088/0264-9381/32/23/234001

Ashtekar, A., Campiglia, M., and Sloan, D. (2009a). Loop Quantum Cosmology and Spin Foams. Phys. Lett. B681, 347-352. doi:10.1016/j.physletb.2009.10.042
Ashtekar, A., Campiglia, M., and Sloan, D. (2010). Casting Loop Quantum Cosmology in the Spin Foam Paradigm. Class. Quant. Grav. 27, 135020. doi:10.1088/0264-9381/27/13/135020

Ashtekar, A., and Campiglia, M. (2012). On the Uniqueness of Kinematics of Loop Quantum Cosmology. Class Quant. Grav. 29, 242001. doi:10.1088/0264-9381/ 29/24/242001

Ashtekar, A., Corichi, A., and Singh, P. (2008). Robustness of Key Features of Loop Quantum Cosmology. Phys. Rev. D 77, 024046. doi:10.1103/physrevd.77. 024046

Ashtekar, A., and Gupt, B. (2017a). Initial Conditions for Cosmological Perturbations. Class. Quan. Grav. 34, 035004. doi:10.1088/1361-6382/aa52d4

Ashtekar, A., and Gupt, B. (2017b). Quantum Gravity in the Sky: Interplay between Fundamental Theory and Observations. Class. Quan. Grav. 34, 014002. doi:10. 1088/1361-6382/34/1/014002

Ashtekar, A., Gupt, B., Jeong, D., and Sreenath, V. (2020). Alleviating the Tension in CMB Using Planck-Scale Physics. Phys. Rev. Lett. 125, 051302. doi:10.1103/ physrevlett.125.051302

Ashtekar, A., and Isham, C. J. (1992). Representations of the Holonomy Algebras of Gravity and Non-abelian Gauge Theories. Class. Quant. Grav. 9, 1433-1467. doi:10.1088/0264-9381/9/6/004

Ashtekar, A., Kaminski, W., and Lewandowski, J. (2009b). Quantum Field Theory on a Cosmological, Quantum Spacetime. Phys. Rev. D79, 064030.

Ashtekar, A., and Lewandowski, J. (2004). Background Independent Quantum Gravity: A Status Report. Class Quant. Grav. 21, R53-R152. doi:10.1088/0264$9381 / 21 / 15 / \mathrm{r} 01$

Ashtekar, A. (2009). Loop Quantum Cosmology: An Overview. Gen. Relativ. Gravit. 41, 707-741. doi:10.1007/s10714-009-0763-4

Ashtekar, A., Pawlowski, T., and Singh, P. (2006a). Quantum Nature of the Big Bang. Phys. Rev. Lett. 96, 141301. doi:10.1103/physrevlett.96.141301

Ashtekar, A., Pawlowski, T., and Singh, P. (2006b). Quantum Nature of the Big Bang: Improved Dynamics. Phys. Rev. D 74, 084003. doi:10.1103/physrevd.74. 084003

Ashtekar, A., and Singh, P. (2004). Loop Quantum Cosmology: A Status Report. Class. Quant. Grav. 28, 213001.

Ashtekar, A., and Singh, P. (2011). Loop Quantum Cosmology: A Status Report. Class. Quant. Grav. 28, 213001. doi:10.1088/0264-9381/28/21/213001

Assanioussi, M., Dapor, A., Liegener, K., and Pawlowski, T. (2018). Emergent de Sitter Epoch of the Quantum Cosmos from Loop Quantum Cosmology. Phys Rev. Lett. 121 (no.8), 081303. doi:10.1103/physrevlett.121.081303

Barrau, A., Cailleteau, T., Grain, J., and Mielczarek, J. (2014). Observational Issues in Loop Quantum Cosmology. Class. Quan. Grav. 31, 053001. doi:10.1088/ 0264-9381/31/5/053001

Barrau, A., Jamet, P., Martineau, K., and Moulin, F. (2018). Scalar Spectra of Primordial Perturbations in Loop Quantum Cosmology. Phys. Rev. D98, 086003.

Bhardwaj, A., Copeland, E. J., and Louko, J. (2019). Inflation in Loop Quantum Cosmology. Phys. Rev. D99, 063520.

Bojowald, M. (2020). Critical Evaluation of Common Claims in Loop Quantum Cosmology. Universe 6, 36. doi:10.3390/universe6030036

Boyanovsky, D., Destri, C., de Vega, H. J., and Sanchez, N. G. (2009). The Effective Theory of Inflation in the Standard Model of the Universe and the CMB+LSS Data Analysis. Int. J. Mod. Phys. A24, 3669-3864. doi:10.1142/ s0217751x09044553

Cai, Y. F., Chen, F., Ferreira, E. G. M., and Quintin, J. (2016). New Model of Axion Monodromy Inflation and its Cosmological Implications. JCAP 1606, 027. doi:10.1088/1475-7516/2016/06/027

Cai, Y. F., Qiu, T. T., Brandenberger, R., and Zhang, X. M. (2009). A Nonsingular Cosmology with a Scale-Invariant Spectrum of Cosmological Perturbations from Lee-Wick Theory. Phys. Rev. D 80, 023511. doi:10.1103/physrevd.80. 023511

Chowdhury, D., Martin, J., Ringeval, C., and Vennin, V. (2019). Inflation after Planck: Judgment Day. Phys. Rev. D100, 083537.

Cline, J. M., Crotty, P., and Lesgourgues, J. (2003). Does the Small CMB Quadrupole Moment Suggest New Physics? JCAP 0309, 010. doi:10.1088/ 1475-7516/2003/09/010

Contaldi, C. R., Peloso, M., Kofman, L., and Linde, A. D. (2003). Suppressing the Lower Multipoles in the CMB Anisotropies. JCAP 307, 002. doi:10.1088/1475 $7516 / 2003 / 07 / 002$ 
Corichi, A., and Singh, P. (2008). Is Loop Quantization in Cosmology Unique? Phys. Rev. D78, 024034.

Craig, D., and Singh, P. (2013). "Consistent Probabilities in Loop Quantum Cosmology”, Class. Quan. Grav 30, 205008. doi:10.1088/0264-9381/30/20/ 205008

Das, S., and Souradeep, T. (2014). Suppressing CMB Low Multipoles with ISW Effect. JCAP 1402, 002. doi:10.1088/1475-7516/2014/02/002

Delabrouille, J., de Bernardis, P., Bouchet, F. R., Achúcarro, A., Ade, P. A. R., Allison, R., et al. CORE Collaboration (2018). Exploring Cosmic Origins with CORE: Survey Requirements and Mission Design. JCAP 04, 014. doi:10.1088/ $1475-7516 / 2018 / 04 / 014$

Di Valentino, E., and Bridle, S. (2018). Exploring the Tension between Current Cosmic Microwave Background and Cosmic Shear Data. Symmetry 10, 585. doi:10.3390/sym 10110585

Di Valentino, E., Melchiorri, A., and Silk, J. (2019). Planck Evidence for a Closed Universe and a Possible Crisis for Cosmology. Nat. Astron 4 (no.2), 196-203. doi:10.1038/s41550-019-0906-9

Domagala, M., and Lewandowski, J. (2004). Black Hole Entropy from Quantum Geometry. Class. Quant. Grav. 21, 5233-5244. doi:10.1088/0264-9381/21/ $22 / 014$

Efstathiou, G. (2020). A Lockdown Perspective on the Hubble Tension(with Comments from the SHOES Team). arXiv:2007.10716v2[astro-ph.CO].

Elizaga Navascués, B., Martín-Benito, M., and Mena Marugán, G. A. (2015). Modified FRW Cosmologies Arising from States of the Hybrid Quantum Gowdy Model. Phys. Rev. D 92 (no.2), 024007. doi:10.1103/physrevd.92. 024007

Engle, J., Hanusch, M., and Thiemann, T. (2017). Uniqueness of the Representation in Homogeneous Isotropic LQC. Commun. Math. Phys. 354 (1), 231-246. [erratum: Commun. Math. Phys. 362, no.2, 759-760 (2018)]. doi:10.1007/ s00220-017-2881-2

Barbero, F., and Perez, A. (2017). "Quantum Geometry and Black Holes," in Loop Quantum Gravity: The First 30 Years ed. A. Ashtekar and J. Pullin (Singapore: World Scientific).

Fernandez-Mendez, M., Mena Marugan, G. A., and Olmedo, J. (2012). Hybrid Quantization of an Inflationary Universe. Phys. Rev. D 86, 024003. doi:10.1103/ physrevd.86.024003

Fialkov, A., and Loeb, A. (2016). Precise Measurement of the Reionization Optical Depth from the Global $21 \mathrm{Cm}$ Signal Accounting for Cosmic Heating. Astrophys. J. 821, 59. doi:10.3847/0004-637x/821/1/59

Fleishchack, C. (2009). Representations of the Weyl Algebra in Quantum Geometry. Commun. Math. Phys. 285, 67-140.

Ganc, J., and Komatsu, E. (2012). Scale-dependent Bias of Galaxies and Mu-type Distortion of the Cosmic Microwave Background Spectrum from Single-Field Inflation with a Modified Initial State. Phys. Rev. D 86, 023518. doi:10.1103/ physrevd.86.023518

Giesel, K. (2017). "Quantum Geometry", in Loop Quantum Gravity: The First 30 Years ed. A. Ashtekar and J. Pullin (Singapore: World Scientific).

Gomar, L. C., Mena-Marugan, G. A., De Blas, D. M., and Olmedo, J. (2017). Hybrid Loop Quantum Cosmology and Predictions for the Cosmic Microwave Background. Phys. Rev. D96, 103528.

Hanany, S., et al.[NASA PICO Collaboration]. (2019). PICO: Probe of Inflation and Cosmic Origins", arXiv:1902.10541[astro-ph.IM].

Handley, W. (2019). Curvature Tension: Evidence for a Closed Universe arXiv: 1908.09139 [astoph-CO].

Jain, R. K., Chingangbam, P., Gong, J.-O., Sriramkumar, L., and Souradeep, T. (2009). Punctuated Inflation and the Low CMB Multipoles. JCAP 0901, 009. doi:10.1088/1475-7516/2009/01/009

Kaminski, W., and Pawlowski, T. (2010). Cosmic Recall and the Scattering Picture of Loop Quantum Cosmology. Phys. Rev. D81, 084027. doi:10.1103/PhysRevD. 81.084027

Kamiński, W., Kolanowski, M., and Lewandowski, J. (2020). Dressed Metric Predictions Revisited. Class. Quant. Grav. 37 (no.9), 095001. doi:10.1088/ 1361-6382/ab7ee0
Kamiński, W. (2012). The Volume Operator in Loop Quantum Cosmology. Phys. Rev. D, 85, 064001

Kofman, L., and Starobinsky, A. A. (1985). Effect of the Cosmological Constant on Large Scale Anisotropies in the Microwave Backbround. Sov. Astron. Lett. 11, 271-274. [Pisma Astron. Zh.11,643(1985)].

Lello, L., Boyanovsky, D., and Holman, R. (2014). Pre-slow Roll Initial Conditions: Large Scale Power Suppression and Infrared Aspects during Inflation. Phys. Rev. D89, 063533. arXiv:1307.4066. doi:10.1103/PhysRevD.89.063533

Lewandowski, J., Okolow, A., Sahlmann, H., and Thiemann, T. (2006). Uniqueness of Diffeomorphism Invariant States on Holonomy-Flux Algebras. Commun. Math. Phys. 267, 703-733. doi:10.1007/s00220-006-0100-7

Lewis, A., and Bridle, S. (2002). Cosmological Parameters from CMB and Other Data: A Monte Carlo Approach. Phys. Rev. D 66, 103511. doi:10.1103/physrevd. 66.103511

Linsefors, L., Cailleteau, T., Barrau, A., and Grain, J. (2013). Primordial Tensor Power Spectrum in Holonomy Corrected Loop Quantum Cosmology. Phys. Rev. D87 (no. 10), 107503

Liu, J., Cai, Y. F., and Li, H. (2012). Evidences for Bouncing Evolution before Inflation in Cosmological Surveys. J. Theor. Phys. 1, 1.

Matsumura, T., et al. (2014). Mission Design of LiteBIRD. J. Low. Temp. Phys. 176, 733. doi:10.1007/s10909-013-0996-1

Meissner, K. (2004). Black Hole Entropy in Loop Quantum Gravity. Class. Quant. Grav. Tetbf21, 5245-5252. doi:10.1088/0264-9381/21/22/015

Olmedo, J., and Alesci, E. (2019). Power Spectrum of Primordial Perturbations for an Emergent Universe in Quantum Reduced Loop Gravity. JCAP 04, 030. doi:10.1088/1475-7516/2019/04/030

Pedro, F. G., and Westphal, A. (2014). Low- CMB Power Loss in String Inflation. JHEP 04, 034. doi:10.1007/JHEP04(2014)034

Penrose, R. (2004). The Road to Reality, (section 28.8). NY: Alfred A Knopf

Perez, A. (2017). "Black Holes in Loop Quantum Gravity". Rept. Prog. Phys. 80, 126901. doi:10.1088/1361-6633/aa7e14

Riess, A. G., Casertano, S., Yuan, W., Macri, L. M., and Scolnic, D. (2019). Large Magellanic Cloud Cepheid Standards Provide a 1\% Foundation for the Determination of the Hubble Constant and Stronger Evidence for Physics beyond CDM. Astrophys. J. 876 (no.1), 85.

Sarkar, D., Huterer, D., Copi, C. J., Starkman, G. D., and Schwarz, D. J. (2011). Missing Power vs Low-L Alignments in the Cosmic Microwave Background: No Correlation in the Standard Cosmological Model. Astropart. Phys. 34, 591. doi:10.1016/j.astropartphys.2010.12.009

Schwarz, D. J., Copi, C. J., Huterer, D., and Starkman, G. D. (2016). CMB Anomalies after Planck. Class. Quant. Grav. 33, 184001. doi:10.1088/0264$9381 / 33 / 18 / 184001$

Singh, P. (2009). Are Loop Quantum Cosmos Never Singular?, Class. Quant. Grav. 26, 125005. doi:10.1088/0264-9381/26/12/125005

Spergel, D. N., et al.[WMAP] (2003). First Year Wilkinson Microwave Anisotropy Probe (WMAP) Observations: Determination of Cosmological Parameters". Astrophys. J. Suppl. 148, 175-194. doi:10.1086/377226

Sreenath, V., Agullo, I., and Bolliet, B. (2019). Computation of Non-Gaussianity in Loop Quantum Cosmology. arXiv:1904.01075[gr-qc]

Zhang, X., Ma, Y., and Artymowski, M. (2013). Loop Quantum Brans-Dicke Cosmology. Phys. Rev. D 87 (no.8), 084024. doi:10.1103/physrevd.87.084024

Conflict of Interest: The authors declare that the research was conducted in the absence of any commercial or financial relationships that could be construed as a potential conflict of interest.

Copyright (c) 2021 Ashtekar, Gupt and Sreenath. This is an open-access article distributed under the terms of the Creative Commons Attribution License (CC BY). The use, distribution or reproduction in other forums is permitted, provided the original author(s) and the copyright owner(s) are credited and that the original publication in this journal is cited, in accordance with accepted academic practice. No use, distribution or reproduction is permitted which does not comply with these terms. 